\title{
Buoyancy-driven motion of a deformable drop toward a planar wall at low Reynolds number
}

\author{
By E. P. ASCOLI, $†$ D. S. DANDY, † AND L. G. LEAL \\ Department of Chemical Engineering, California Institute of Technology, \\ Pasadena, CA 91125, USA
}

(Received 17 February 1989)

\begin{abstract}
The slow viscous motion of a deformable drop moving normal to a planar wall is studied numerically. In particular, a boundary integral technique employing the Green's function appropriate to a no-slip planar wall is used. Beginning with spherical drop shapes far from the wall, highly deformed and 'dimpled' drop configurations are obtained as the planar wall is approached. The initial stages of dimpling and their evolution provide information and insight into the basic assumptions of filmdrainage theory.
\end{abstract}

\section{Introduction}

The slow viscous motion of deformable drops is of interest in processes such as coalescence, emulsion formation, mixing and separation. In real systems bounding surfaces are always present. However, little is known about the effects of such bounding surfaces on drop motions and shapes. Haberman \& Sayre (1958) obtained approximate solutions for a spherical drop travelling in steady motion along the axis of a tube. Bart (1968) solved the problem of the slow unsteady settling of a fluid sphere toward a flat fluid interface. However, in each of these analyses, the assumption of spherical (or near-spherical) shape is a fundamental limitation.

A multitude of investigators have theoretically considered the effects of a planar wall on drops and bubbles when the separation distance between the wall and the drop or bubble is very small. In these investigations, the focus has been on the dynamics of the thin film between the bounding wall and the drop or bubble, without attempting to resolve the dynamics that lead to the thin-film configuration. Experimentally, (Hartland 1967, 1969; Platinakov 1964) 'dimpled' configurations (i.e. relatively thin films that are thinner near the rim than at the centre) have been observed for a wide range of parameters. Theoretically, the analyses of this configuration have been based upon the lubrication approximation in an attempt to model the dynamics of film-drainage. Unfortunately, many of the simplifying assumptions have been ad hoc, and largely unmotivated. The most important of those assumptions are listed below.

(i) The film has been assumed sufficiently thin that the lubrication approximation is valid (Frankel \& Mysels 1962; Hartland 1969; Dimitrov \& Ivanov 1978; Lin \&

$\dagger$ Present address: Rockwell International, Rocketdyne Division M/S WC75, 6633 Canoga Avenue, Canoga Park, CA 91303, USA.

† Present address: Div. 8363, Sandia National Laboratories, PO Box 969, Livermore, CA 94550, USA.

$\S$ Present address : Department of Chemical and Nuclear Engineering, University of California, Santa Barbara, CA 93106, USA. 
Slattery 1982). Specifically, if $h$ is a measure of the film thickness and $R$ is a measure of the radial extent of the film then $h / R \ll 1$. This is the key assumption underlying film-drainage theory. In particular, it has been implicitly assumed that the lubrication approximation includes the necessary physics to predict the onset and evolution of dimpling.

(ii) Viscous effects in the drop have generally been neglected. In particular, focus has been placed on the two limiting cases of 'fully mobile' (zero tangential stress) and 'fully immobile' interfaces (no-slip applied at the fluid-fluid interface), (Frankel \& Mysels 1962; Hartland 1969; Dimitrov \& Ivanov 1978; Lin \& Slattery 1982).

(iii) Gravitational effects in the film have frequently been neglected (Hartland 1969; Dimitrov \& Ivanov 1978; Lin \& Slattery 1982).

(iv) In addition to assumption (i), a wide variety of geometrical assumptions have been made:

(a) The film has been assumed to be nearly a plane parallel to the wall (Dimitrov \& Ivanov 1978).

(b) 'Dimpled' configurations have been assumed as initial film shapes. For instance, the dimple has been assumed to consist of two parabolas with the radius of curvature at the apex varying with time in the central parabola and constant in the peripheral parabola (Hartland \& Robinson 1977).

(c) The drop has been assumed nearly spherical outside of the film region (Lin \& Slattery 1982; Dimitrov \& Ivanov 1978).

(d) At the edge of the film, geometrical details of drop shape have been assumed nearly invariant with time (Dimitrov \& Ivanov 1978; Lin \& Slattery 1982).

The list of thin-film investigations referenced above is by no means complete but it is illustrative. It is quite surprising, after literally dozens of papers based upon the film-drainage model that no previous attempt seems to have been made to explore the legitimacy of the many assumptions that have been used in its development.

In this paper we present an efficient numerical scheme for determining the buoyancy-driven motion of a deformable viscous drop normal to a planar no-slip surface. The complete time evolution of drop shape from spherical far from the wall to highly deformed near the wall is studied. No limitation on film thickness is made. Viscous effects in the drop phase are not neglected. Gravity is not neglected. The only geometrical assumptions are that the motion is axisymmetric and the drop is initially spherical far from the wall.

The primary goal of the study is to explore the film-drainage assumptions listed above using a complete numerical scheme. In addition, this study is intended to provide information and insight into the details and mechanism behind dimple formation. In principle, of course, these goals could also be approached from an experimental point of view or, in the final stages of film dynamics, via the development of a complete asymptotic theory in which the flow and drop shape in the film region are coupled with the flow and shape in the rest of the domain. However, in spite of considerable effort, a complete asymptotic description has so far eluded us and, in any case, such a theory could not provide a basis to explore the formation of the thin film, only its dynamics after formation. Further, experiments with sufficient resolution to determine velocity and pressure fields within the film or even the details of the film geometry are very difficult, if not impossible. Hence, in our view, a direct comparison with exact numerical results provides the most logical and productive basis for examining the many assumptions of existing film-drainage models. Since the current scheme is subject to numerical errors as well as time 
limitations, the results obtained pertain strictly to the initial stages of thin film formation. Drop shapes obtained with the current numerical scheme may potentially be used as starting configurations for thin-film analytic theories.

The technique that we use is based on the well-known boundary integral method that has already been used successfully in a variety of applications. Youngren \& Acrivos applied the method to calculate the slow viscous flow past a single solid particle (1975). Later, Youngren \& Acrivos (1976) applied the technique to calculate the steady-state deformation of an inviscid drop in an extensional flow, and Rallison \& Acrivos (1978) considered viscous drops in an extensional flow. Lee \& Leal (1982) and later Geller, Lee \& Leal (1986) used the boundary integral method to calculate the motion of a solid sphere normal to a deformable interface. Recently, Chi \& Leal (1989) considered the motion of a deformable drop normal to a deformable fluid-fluid interface, also using the boundary integral method. The current application of the method differs from these previous studies in the choice of the so-called fundamental solution of the Stokes' system. Here we employ a Green's function appropriate for systems involving an infinite, planar wall. The concept of employing a Green's function in a boundary integral or singularity formulation has been pursued successfully by a variety of researchers. Zick \& Homsy (1982) employed the Green's function appropriate to a periodic array of fixed spheres. Pozrikidis (1987) examined two-dimensional, creeping, channel flow using the appropriate Green's function. TranCong \& Phan-Thien (1989) used Green's function concepts to treat multiparticle systems undergoing Stokes flow. Ascoli, Dandy \& Leal (1989) determined the hydrodynamic resistance on a solid particle in creeping flow moving in the presence of a planar wall using the same Green's function that will be employed here.

\section{Formulation}

\subsection{Equations and boundary conditions}

We consider the slow motion of a deformable drop normal to a no-slip, infinite, planar surface (figure 1a). Fluid 1, which comprises the drop, and fluid 2, in which the drop is suspended are both assumed to be Newtonian with constant density. In addition, the fluids are assumed to be immiscible with an interface characterized by a constant surface tension. All motions and deformations are assumed axisymmetric about the $z$-axis, which passes through the drop centre and is normal to the planar wall. Gravity is assumed to act along the $z$-axis, toward the wall if fluid 1 is more dense than fluid 2, or away from the wall if fluid 1 is less dense than fluid 2 . The analysis presented is based upon the creeping motion approximation in which the intertial terms in the equations of motion are neglected entirely. The corresponding small Reynolds numbers of the system are

$$
R e_{i}=\frac{U a}{\nu_{i}} \ll 1 \quad(i=1,2) .
$$

The characteristic length, $a$, is the radius of a sphere of equal volume and $v_{1}, v_{2}$ are the respective kinematic viscosities of fluid 1 and fluid 2 . The characteristic velocity, $U$, is the terminal velocity of an equal volume spherical drop in the absence of a wall and is given by the Hadamard (1911) and Rybczynski (1911) result

$$
U=\frac{1}{3} \frac{a^{2} g}{v_{2}}\left|\frac{\rho_{1}}{\rho_{2}}-1\right|\left(\frac{1+\lambda}{1+\frac{3}{2} \lambda}\right),
$$


(a)

\section{Fluid 2}

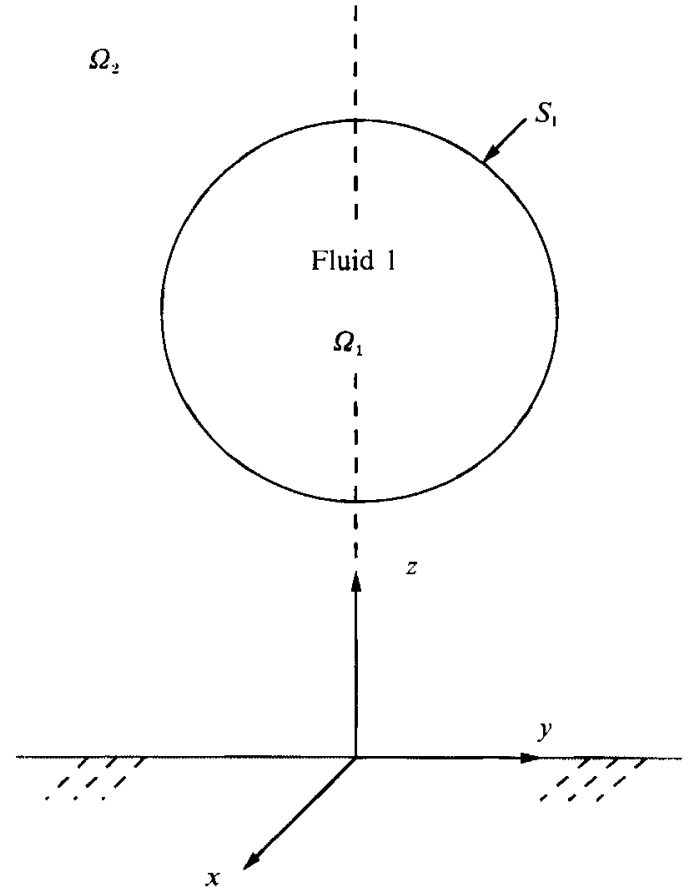

(b)

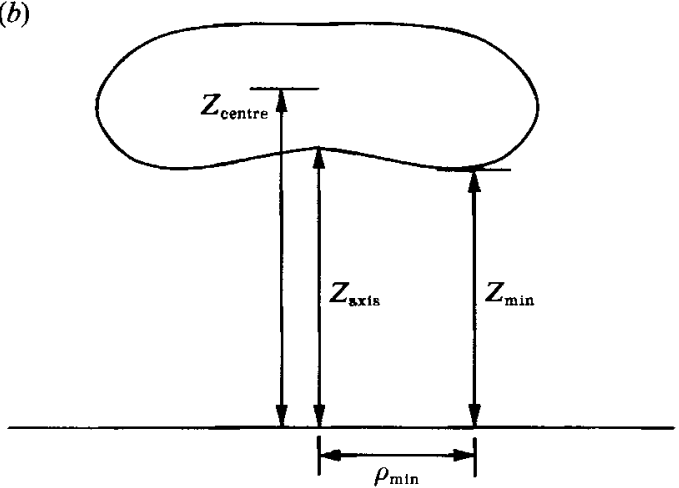

Figure 1. (a) Schematic of the problem. (b) An illustration of the quantities

$$
Z_{\text {centre }}, Z_{\text {axis }}, Z_{\min } \text {, and } \rho_{\text {min }} \text {. }
$$

where $\lambda=\mu_{1} / \mu_{2}$ is the ratio of dynamic viscosities while $\rho_{1}$ and $\rho_{2}$ are the respective fluid densities. The governing Stokes equations are then:

$$
0=-\nabla p_{i}+\nabla^{2} u_{i}, \quad 0=\nabla \cdot u_{i} \quad(i=1,2) .
$$

Here, velocities are made dimensionless with $U$ and lengths with $a$. Pressures and stresses are made dimensionless with $\mu_{i} U / a$. The boundary conditions are

$$
\begin{array}{ll}
u_{2} \rightarrow 0 & \text { as }\|x\| \rightarrow \infty, \\
u_{2}=0 & \text { for } z=0
\end{array}
$$


and at the drop interface, $x \in S_{\mathrm{I}}$ :

$$
\begin{aligned}
& u_{1}=u_{2}=u^{\text {def }}, \\
& \left(\lambda n \cdot T^{(1)}-n \cdot T^{(2)}\right)=-\frac{n}{C a} \nabla \cdot n-n z\left\{3 \frac{\left(1+\frac{3}{2} \lambda\right)}{(1+\lambda)}\right\}^{\text {def }}=\mathscr{F} .
\end{aligned}
$$

The vector $n$ is the outer normal to the drop surface. The pressures $p_{1}$ and $p_{2}$ are modified pressures $\uparrow$ which represent the pressure contribution in the fluid due solely to motion. Gravity enters the problem through (5) via the term in curly brackets. Here,

is the capillary number.

$$
C a=\mu_{2} U / \gamma
$$

Given a drop configuration at dimensionless time $t$, (time made dimensionless with respect to $a / U$ ) equations (1)-(5) determine the instantaneous velocity field. The drop surface, $S_{I}$, is assumed to deform in accord with the instantaneous interface velocity field, thus, determining a new drop configuration. In this manner, the time evolution of the drop is determined. The kinematic condition used to deform the drop surface is

$$
\frac{\mathrm{d} x_{\mathrm{s}}}{\mathrm{d} t}=\boldsymbol{n}\left(\boldsymbol{x}_{\mathrm{s}}\right)\left(\boldsymbol{n}\left(\boldsymbol{x}_{\mathrm{s}}\right) \cdot u^{\mathrm{I}}\left(\boldsymbol{x}_{\mathrm{s}}\right)\right)
$$

In other words, the drop surface is deformed pointwise along the normal with the normal projection of the surface velocity.

Ascoli, Dandy \& Leal (1989) presented the boundary integral formulation appropriate for the case where an infinite, no-slip, planar wall is present. There, the formulation was applied to determine the hydrodynamic resistances on solid particles in creeping flow near a no-slip planar wall. The extension to the two-fluid case is straightforward; thus, we present only the results. Details of the derivation can be found in Ascoli (1988).

System (1) and the corresponding boundary conditions when recast in boundary integral form and evaluated at a point $x_{\mathrm{s}} \in S_{\mathrm{I}}$ yield:

$$
\begin{gathered}
\frac{1}{2}\left(u^{\mathrm{I}}\left(x_{\mathrm{s}}\right)\right)_{j}=+\int_{S_{\mathrm{I}}} \Sigma^{j}\left(x_{\mathrm{s}}, \xi\right) \cdot u^{\mathrm{I}}(\xi) \cdot n \mathrm{~d} S_{\xi}-\int_{S_{\mathrm{I}}} v^{j}\left(x_{\mathrm{s}}, \xi\right) \cdot f^{(1)}(\xi) \mathrm{d} S_{\xi} \\
-\frac{1}{2}\left(u^{\mathrm{I}}\left(x_{\mathrm{s}}\right)\right)_{j}=-\frac{\lambda-1}{\lambda+1} \int_{S_{\mathrm{I}}} \Sigma^{j}\left(x_{\mathrm{s}}, \xi\right) \cdot u^{\mathrm{I}}(\xi) \cdot n \mathrm{~d} S_{\xi}+\frac{1}{(\lambda+1)} \int_{S_{\mathrm{I}}} v^{j}(x, \xi) \cdot \mathscr{F} \mathrm{d} S_{\xi},
\end{gathered}
$$

where $f^{(i)}=n \cdot T^{(i)}$.

The wall Green's function kernels $v^{j}(x, \xi), q^{j}(x, \xi)$ and $\Sigma^{j}(x, \xi)$ and discussed by Blake (1971) and stem from the work of Oseen (1927) and Odqvist (1930). They are given by:

$$
v_{i}^{j}=\hat{v}_{i}^{j}+\hat{\hat{v}}_{i}^{j}, \quad q^{j}=\hat{q}^{j}+\hat{\hat{q}}^{j}, \quad \Sigma_{i k}^{j}=\hat{\Sigma}_{i k}^{j}+\hat{\Sigma}_{i k}^{j},
$$

where

$$
\hat{v}_{i}^{j}=-\frac{1}{8 \pi}\left\{\frac{\delta_{i j}}{r}+\frac{r_{i} r_{j}}{r^{3}}\right\}, \quad \hat{q}^{j}=\frac{1}{4 \pi}\left\{\frac{r_{j}}{r^{3}}\right\}, \quad \hat{\Sigma}_{i k}^{j}=\frac{3}{4 \pi}\left\{\frac{r_{k} r_{i} r_{j}}{r^{5}}\right\},
$$

$\dagger p_{i}=P_{i} \pm \rho g z a^{2} / \mu_{i} U$ where $P_{i}$ is absolute pressure and the sign is determined by the direction in which gravity acts. 
and

$$
\begin{aligned}
\hat{\hat{v}}_{i}^{j}= & -\frac{1}{8 \pi}\left\{-\frac{\delta_{i j}}{R}-\frac{R_{i} R_{j}}{R^{3}}\right. \\
& +2 x_{3} \Delta_{j}\left[x_{3}\left(\frac{\delta_{i j}}{R^{3}}-\frac{3 R_{i} R_{j}}{R^{5}}\right)+\frac{\delta_{i 3}}{R^{3}} R_{j}\right. \\
& \left.\left.-\frac{1}{R^{3}}\left(\delta_{i j} R_{3}+R_{i} \delta_{3 j}\right)+\frac{3 R_{i} R_{j} R_{3}}{R^{5}}\right]\right\}, \\
\hat{\hat{q}}^{j}= & \frac{1}{4 \pi}\left[-\frac{R_{j}}{R^{3}}-2 x_{3} \Delta_{j}\left(\frac{\delta_{3 j}}{R^{3}}-\frac{3 R_{j} R_{3}}{R^{5}}\right)\right],
\end{aligned}
$$

$\hat{\Sigma}_{i k}^{j}=\frac{3}{4 \pi}\left\{-\frac{R_{i} R_{j} R_{k}}{R^{5}}-2 x_{3} \Delta_{j}\left[-\frac{x_{3}}{R^{5}} \delta_{i k} R_{j}+\frac{\xi_{3}}{R^{5}}\left(R_{i} \delta_{j k}+\delta_{i j} R_{k}\right)+\frac{R_{i} \delta_{3 j} R_{k}}{R^{5}}-\frac{5 R_{i} R_{j} R_{k} \xi_{3}}{R^{7}}\right]\right\}$.

where $\quad r=\left(\xi_{1}-x_{1}, \xi_{2}-x_{2}, \xi_{3}-x_{3}\right)^{T}, \quad R=\left(\xi_{1}-x_{1}, \xi_{2}-x_{2}, \xi_{3}+x_{3}\right)^{T}, r=\left[\left(\xi_{1}-x_{1}\right)^{2}+\right.$ $\left.\left(\xi_{2}-x_{2}\right)^{2}+\left(\xi_{3}-x_{3}\right)^{2}\right]^{\frac{1}{2}}, R=\left[\left(\xi_{1}-x_{1}\right)^{2}+\left(\xi_{2}-x_{2}\right)^{2}+\left(\xi_{3}-x_{3}\right)^{2}\right]^{\frac{1}{2}}$, and $R_{3}=\xi_{3}+x_{3}$. The quantity $\Delta_{j}$ has value +1 for $j=1,2$ and -1 for $j=3$. Here $\hat{v}_{i}^{j}, \hat{q}^{j}$ and $\hat{\Sigma}_{i k}^{j}$ is the classical 'fundamental solution' to Stokes system employed by previous researchers. Physically, $\hat{v}_{i}^{j}$ is the $i$ th component of the velocity at $\xi$ due to a unit force, or Stokeslet, applied at the point $\boldsymbol{x}$ in the direction $e_{j}$. The quantity $-\hat{q}^{j}$ is the corresponding pressure. The Green's function (9) can be viewed as the fundamental solution to Stokes equations plus additional terms to account for the presence of the planar wall.

Given a drop shape, (7) and (8) when numerically discretized allow computation of $f^{(i)}$ and $u^{\mathrm{I}}$. Velocity and pressure fields interior and exterior to the drop may then be calculated using:

$$
\begin{gathered}
(-1)^{i}\left(u_{i}(x)\right)_{j}=\int_{S_{\mathrm{I}}}\left(n \cdot T^{(i)}(\xi) \cdot v^{j}(x, \xi)-n \cdot \Sigma^{j}(x, \xi) \cdot u^{\mathrm{I}}(\xi)\right) \mathrm{d} S_{\xi} \\
p_{i}(x)-\bar{p}_{i}=-\int_{S_{\mathrm{I}}}\left(f^{(i)}(\xi) \cdot \Psi(x, \xi)-n \cdot \Phi(x, \xi) \cdot u^{\mathrm{I}}(\xi)\right) \mathrm{d} S_{\xi} \\
\left(x \in \Omega_{i}, i=1,2\right)
\end{gathered}
$$

The terms $\bar{p}_{i}$ are constants of integration with $\bar{p}_{2}$ being the pressure as $z \rightarrow \infty$. The kernels $\boldsymbol{\Psi}$ and $\boldsymbol{\Phi}$ are given by

$$
\begin{gathered}
\Psi_{k}(x, \xi)=\frac{1}{4 \pi}\left[\frac{r_{k}}{r^{3}}-\frac{R_{k}}{R^{3}}+\frac{2 x_{3} \delta_{k 3}}{R^{3}}+\frac{6 \xi_{3} R_{k} R_{3}}{R^{5}}\right] \\
\Phi_{i k}(x, \xi)=\frac{1}{2 \pi}\left[\frac{\delta_{i k}}{r^{3}}-\frac{3 r_{i} r_{k}}{r^{5}}+\frac{\delta_{i k}}{R^{3}}+\frac{3 R_{i} R_{k}}{R^{5}}-\frac{6 x_{3} R_{3} \delta_{i k}}{R^{5}}\right. \\
\left.+\frac{6 \xi_{3}\left(\delta_{i 3} R_{k}+\delta_{k 3} R_{i}\right)}{R^{5}}-\frac{30 \xi_{3} R_{3} R_{i} R_{k}}{R^{7}}\right] .
\end{gathered}
$$

There are several ways in which the drop surface may be represented. One possibility is to define the surface as $(\rho(z), z)$ for $\theta \in[0,2 \pi)$, where $\rho=\left(x^{2}+y^{2}\right)^{\frac{1}{2}}$ and $\theta$ are cylindrical coordinates. However, this representation implicitly limits the types of surfaces which can be considered to those for which $\rho$ is a single-valued function of $z$. Alternately, a spherical coordinate system could be employed, with $\rho_{\text {sphere }}=$ 
$\left(x^{2}+y^{2}+z^{2}\right)^{\frac{1}{2}}$ and polar angle $\phi$ measured from the $z$-axis, to give a representation $\left(\rho_{\text {sphere }}(\phi), \phi\right)$ for $\theta \in[0,2 \pi)$ and $\phi \in[0, \pi)$. Again this representation is limited to those surfaces which yield single-valued functions $\rho_{\text {sphere }}(\phi)$. An equally simple, but more general representation which avoids this potential problem is to parametrically define the surface as $(\rho(s), z(s))$ for $\theta \in[0,2 \pi)$, where $s$ is a parametric independent variable lying in the interval $\left[s_{0}, s_{f}\right]$. This is the approach taken here. In particular $s$ is chosen to be normalized arclength.

In terms of the functions $\rho(s)$ and $z(s)$ which define the surface, the components of the unit normal to the surface are

and the curvature is

$$
n_{\rho}=\frac{\dot{z}}{\left(\dot{\rho}^{2}+\dot{z}^{2}\right)^{\frac{1}{2}}}, \quad n_{z}=-\frac{\dot{\rho}}{\left(\dot{\rho}^{2}+\dot{z}^{2}\right)^{\frac{1}{2}}}
$$

$$
\boldsymbol{\nabla} \cdot \boldsymbol{n}=\frac{\dot{z}}{\rho\left(\dot{\rho}^{2}+\dot{z}^{2}\right)^{\frac{1}{2}}}+\frac{\dot{\rho} \ddot{z}-\ddot{\rho} \dot{z}}{\left(\dot{\rho}^{2}+\dot{z}^{2}\right)^{\frac{3}{2}}}
$$

where dots indicate differentiation with respect to the variable $s$.

Due to axisymmetry, (7) and (8) can be further simplified. The dependence of the integrands on $\theta$ is known and integration with respect to $\theta$ can be performed. Although the resulting analytic integration yields quite complex expressions, the net result is a reduction of the integration domain from two dimensions to one. The results are

$$
\begin{gathered}
\frac{1}{2}\left(\begin{array}{l}
u_{\rho}^{\mathrm{I}}(x) \\
u_{z}^{\mathrm{I}}(x)
\end{array}\right)=+\int_{s_{0}}^{s_{f}} \boldsymbol{P}(\boldsymbol{x}, \xi) \cdot\left(\begin{array}{l}
u_{\rho}^{\mathrm{I}}(\xi) \\
u_{z}^{\mathrm{I}}(\xi)
\end{array}\right) \mathrm{d} \hat{s}-\int_{s_{0}}^{s_{f}} \boldsymbol{Q}(\boldsymbol{x}, \xi) \cdot\left(\begin{array}{l}
f_{\rho}^{(1)}(\xi) \\
f_{z}^{(1)}(\xi)
\end{array}\right) \mathrm{d} \hat{s} \\
\frac{1}{2}\left(\begin{array}{l}
u_{\rho}^{\mathrm{I}}(x) \\
u_{z}^{\mathrm{I}}(x)
\end{array}\right)=\frac{\lambda-1}{\lambda+1} \int_{s_{0}}^{s_{f}} \boldsymbol{P}(\boldsymbol{x}, \xi) \cdot\left(\begin{array}{l}
u_{\rho}^{\mathrm{I}}(\xi) \\
u_{z}^{\mathrm{I}}(\xi)
\end{array}\right) \mathrm{d} \hat{s}-\frac{1}{(\lambda+1)} \int_{s_{0}}^{s_{f}} \boldsymbol{Q}(\boldsymbol{x}, \xi) \cdot\left(\begin{array}{l}
\mathscr{F}_{\rho}(\xi) \\
\mathscr{F}_{z}(\xi)
\end{array}\right) \mathrm{d} \hat{s} \quad\left(x \in S_{\mathrm{I}}\right) \\
\mathrm{d} \hat{s}=\mathrm{d} s \rho\left(\dot{\rho}^{2}+\dot{z}^{2}\right)^{\frac{1}{2}}
\end{gathered}
$$

Expressions for the matrices $\boldsymbol{P}$ and $\boldsymbol{Q}$ may be found in Ascoli (1988).

This system provides a relationship between the velocity of the fluid 1 /fluid 2 interface, the interfacial stress and the interfacial curvature. Given the set of surface variables $\rho, z, \dot{\rho}, \dot{z}, \ddot{\rho}$, and $\ddot{z}$ at time $t$, this system allows calculation of the instantaneous interfacial velocity and stress. Once the interfacial velocity is determined, the interface is deformed in accordance with the kinematic condition (6). At any instant, once the interfacial velocities and stresses are known, velocities and pressures in the interior of the fluid domain may be calculated from the $\theta$-integrated forms of $(10)$.

\subsection{Implementation}

System $(15,16)$ may be discretized and numerically solved. The approach taken here employs the method of Krylov-Bogoliubov (Kantorovich \& Krylov 1963). Specifically, the interface arc $(\rho(s), z(s)), s \in\left[s_{0}, s_{f}\right]$ is divided into $N$ elements. This is accomplished by dividing the interval $\left[s_{0}, s_{f}\right]$ into subintervals, $\Delta s_{j}$, with centres $s_{j}(j=1, \ldots, N)$. Each parametric value, $s_{j}$, corresponds to a point on the arc $x_{j}=\left(\rho\left(s_{j}\right)\right.$, $\left.z\left(s_{j}\right)\right)$ and each $\Delta s_{j}$ corresponds to a segment or element of the arc. The elements are assumed to be sufficiently small so that the local normal tractions $f_{\rho}^{(1)}, f_{z}^{(1)}$ and 
velocities $u_{\rho}^{\mathrm{I}}, u_{z}^{\mathrm{I}}$ may be assumed constant within each element. The resulting discretized system is:

$$
\begin{aligned}
\frac{1}{2}\left(\begin{array}{l}
u_{\rho}^{\mathrm{I}}\left(\boldsymbol{x}_{i}\right) \\
u_{z}^{\mathrm{I}}\left(\boldsymbol{x}_{i}\right)
\end{array}\right) \simeq & +\sum_{j=1}^{N}\left\{\left[\int_{\Delta s_{j}} \boldsymbol{P}\left(\boldsymbol{x}_{i}, \xi\right) \mathrm{d} \hat{s}\right] \cdot\left(\begin{array}{l}
u_{\rho}^{\mathrm{I}}\left(\boldsymbol{x}_{j}\right) \\
u_{z}^{\mathrm{I}}\left(\boldsymbol{x}_{j}\right)
\end{array}\right)\right\} \\
& -\sum_{j=1}^{N}\left\{\left[\int_{\Delta s_{j}} \boldsymbol{Q}\left(\boldsymbol{x}_{i}, \xi\right) \mathrm{d} \hat{s}\right] \cdot\left(\begin{array}{l}
f_{\rho}^{(1)}\left(\boldsymbol{x}_{j}\right) \\
f_{z}^{(1)}\left(\boldsymbol{x}_{j}\right)
\end{array}\right)\right\} \\
\frac{1}{2}\left(\begin{array}{l}
u_{\rho}^{\mathrm{I}}\left(\boldsymbol{x}_{i}\right) \\
u_{z}^{\mathrm{I}}\left(\boldsymbol{x}_{i}\right)
\end{array}\right) \simeq & \frac{\lambda-1}{\lambda+1} \sum_{j=1}^{N}\left\{\left[\int_{\Delta s_{j}} \boldsymbol{P}\left(\boldsymbol{x}_{i}, \xi\right) \mathrm{d} \hat{s}\right] \cdot\left(\begin{array}{l}
u_{\rho}^{\mathrm{I}}\left(\boldsymbol{x}_{j}\right) \\
u_{z}^{\mathrm{I}}\left(\boldsymbol{x}_{j}\right)
\end{array}\right)\right\} \\
& -\frac{1}{\lambda+1} \sum_{j=1}^{N}\left\{\left[\int_{\Delta s_{j}} \boldsymbol{Q}\left(\boldsymbol{x}_{i}, \xi\right) \cdot\left(\begin{array}{l}
\mathscr{F}_{\rho}\left(\boldsymbol{x}_{j}\right) \\
\mathscr{F}_{z}\left(\boldsymbol{x}_{j}\right)
\end{array}\right) \mathrm{d} \hat{s}\right]\right\} \quad(1 \leqslant i \leqslant N)
\end{aligned}
$$

Given the details of the initial shape and curvature, each coefficient

$$
\int_{\Delta s_{j}} \boldsymbol{P}\left(\boldsymbol{x}_{i}, \xi\right) \mathrm{d} \hat{s}, \quad \int_{\Delta s_{j}} \boldsymbol{Q}\left(\boldsymbol{x}_{i}, \xi\right) \mathrm{d} \hat{s}
$$

and inhomogeneous term

$$
\int_{\Delta \varepsilon_{j}} \boldsymbol{Q}\left(\boldsymbol{x}_{i}, \xi\right) \cdot\left(\begin{array}{l}
\mathscr{F}_{\rho}\left(\boldsymbol{x}_{j}\right) \\
\mathscr{F}_{z}\left(\boldsymbol{x}_{j}\right)
\end{array}\right) \mathrm{d} \hat{s} \quad \text { for } j \neq i
$$

can be easily evaluated by standard numerical integration schemes. Here Gaussian quadrature was used. For $j=i$ and $s=s_{i}$ then $\xi(s)=\boldsymbol{x}_{i}$, and the functions $\boldsymbol{P}$ and $\boldsymbol{Q}$ become unbounded. In this case, approximate analytic treatment of the singular integrand is performed to include accurately the singular contribution (Ascoli 1988).

Equation (18) is then a $2 N \times 2 N$ linear system in the unknown velocities which is easily solved by standard methods. If desired, the unknown surface forces may then be determined by solving (17). Once a shape and velocity distribution at time $t$ is known, an explicit discretization of (6) is used to determine a new shape and subsequent velocity distribution. This determines a new set of surface locations $\left(\rho\left(s_{j}\right), z\left(s_{j}\right)\right)$ for $j=1, \ldots, N$ at time $t+\Delta t$. Cubic splines are used to determine $\rho(s), \dot{\rho}(s)$, $\ddot{\rho}(s), z(s), \dot{z}(s), \ddot{z}(s)$ for $s \in\left[s_{0}, s_{f}\right]$. The $t+\Delta t$ velocities are then calculated using these shape details and the process is repeated, marching forward in time.

\section{Numerical results}

\subsection{Preliminary testing}

As an initial test of the wall Green's function approach, drag calculations were performed on solid ellipsoids moving axisymmetrically toward the wall. Agreement with existing theory was excellent. For further details the reader is referred to Ascoli et al. (1989). Calculations were performed on a spherical drop located far (1000 radii) from the wall. Comparison with the results of Hadamard (1911) and Rybczynski (1911) was excellent (Ascoli 1988).

The calculated drag, found by integrating the local surface forces $\left(f_{\rho}, f_{z}\right)$, theoretically should equal the constant applied buoyant force. Thus, the error in this computed value may be used as a check of the accuracy of the technique for all ranges of drop to wall distance. Using calculated drag error as a guide, it was determined that 32 surface elements were adequate for the range of parameters considered.

In the course of the computations, errors in drop volume, though extremely small 
at each given time/shape calculation, were found to accumulate. Typically a calculation using 32 elements beginning with a spherical drop shape 15 radii away and terminating with a highly deformed drop within one undeformed radius of the wall was found to have a total accumulated error in volume of the order of $5 \%$. With a timestep of 0.005 and a run time of approximately 20 dimensionless time units this corresponds to roughly $0.00125 \%$ error in volume per iteration. Although slightly improved accuracy in volume was obtained by using 40 and 48 elements, the additional computational time did not warrant the use of more than 32 elements. Instead, for all the results presented below, a periodic renormalization of the length scaling was performed during the computations to maintain a constant drop volume.

A test was performed to determine the distance from the wall at which the assumption of a spherical starting shape is reasonable. A spherical drop was released with centre of mass at an initial distance of 25 undeformed radii from the wall (i.e. $Z_{\text {centre }}=25$ ) and the calculations were continued until $Z_{\text {centre }}=15$. The centre of mass velocity, $V_{\text {centre }}$, differed from the normalized value of one by as much as $6.4 \%$ for $C a=3, \lambda=0.3$. However, the maximum deviation in radius from one undeformed radii

$$
\max \left(\left[\left(z_{i}-Z_{\text {centre }}\right)^{2}+\rho_{i}^{2}\right]-1\right)^{\frac{1}{2}}
$$

for this case was less than $2 \%$. For $C a=1$ and $C a=0.3$, the maximum deviation from a constant radius was less than 0.85 and $0.25 \%$, respectively, for the $\lambda$ values considered. These results indicate that a starting position of $Z_{\text {centre }}=15$ with a spherical initial shape is an excellent assumption for $C a=0.3$ and 1 , and a reasonable assumption for $C a=3$.

Hartland (1969) performed a series of experiments involving drops of golden syrup containing potassium iodide falling under the influence of gravity through sextol phthalate towards a planar wall. Although the intent of his experiments was to study the later stages of dimple formation when the golden syrup drops are extremely close to the wall, his photographic results for the early stages of dimple formation provide experimental details of the gross shape of the entire drop which may be compared with the current numerics. Hartland's physical parameters for the high viscosity golden syrup drop $\left(\rho_{1}=1.580 \mathrm{~g} / \mathrm{cm}^{3}, \rho_{2}=1.069 \mathrm{~g} / \mathrm{cm}^{3}, \mu_{1}=175 \mathrm{P}, \mu_{2}=137 \mathrm{P}, \gamma=\right.$ 23.6 dyne $/ \mathrm{cm}$, with drop volume of $0.25 \mathrm{~cm}^{3}$ ) give $U=0.234 \mathrm{~cm} / \mathrm{s}, C a=1.358, \lambda=$ 1.478 , and $R e_{1}=8.25 \times 10^{-4}, R e_{2}=7.13 \times 10^{-4}$. Numerical results are shown in figure 2 for the case of $C a=1, \lambda=1$ with $Z_{\text {axis }}=0.155$. This is the smallest value of $Z_{\text {axis }}$ obtained numerically for these parameters. Hartland's photograph corresponds to $Z_{\text {axis }}$ between 0.153 and 0.128 . The agreement in the shape is exceptional.

\subsection{Results}

The calculations presented here were carried out using thirty-two elements with a timestep of 0.005 . Each run was terminated when the calculated drag differed from the theoretical value by more than $2 \%$. Results are reported for $\lambda$ of 3,1 and 0.3 , with $C a$ equal to 3,1 and 0.3 for each $\lambda$. For all calculations the initial shape was taken to be spherical, with the sphere centre at 15 radii from the wall.

The calculated drop shapes are shown in figure 3 for all nine combinations of $\lambda$ and $C a$. The elapsed time between each shape shown is 1 dimensionless time unit (i.e. the time increment required for the undeformed drop in an unbounded fluid to translate a distance equal to its own radius).

Dimpling was observed for all values of $\mathrm{Ca}$ and $\lambda$ considered. Shape evolution naturally divides into two regimes: evolution prior to the point of dimpling and evolution after a dimple has formed. The transition configurations, i.e. the 


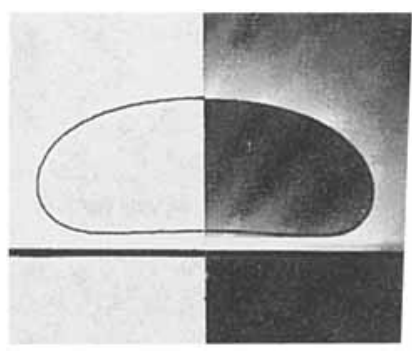

Figure 2. Comparison with the experimental results of Hartland (1969). The left-hand half of the figure corresponds to the numerical results for $C a=1, \lambda=1$, and $Z_{\mathrm{axis}}=0.155$. The right-hand half of the figure is an experimental photograph for $C a=1.358, \lambda=1.478$ and a $Z_{\text {axis }}$ value between 0.153 and 0.128 .

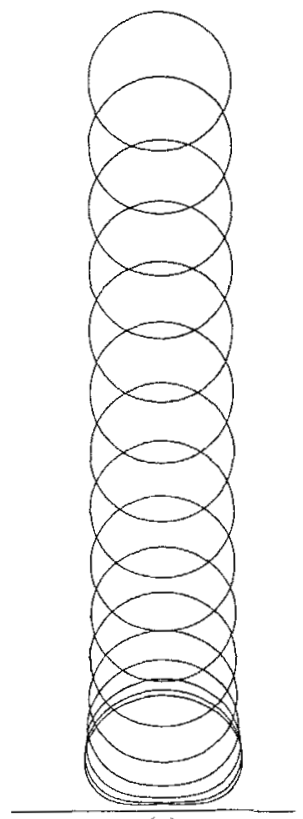

(a)

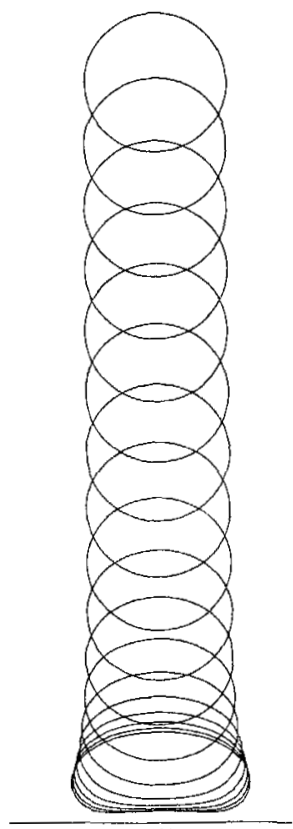

(b)

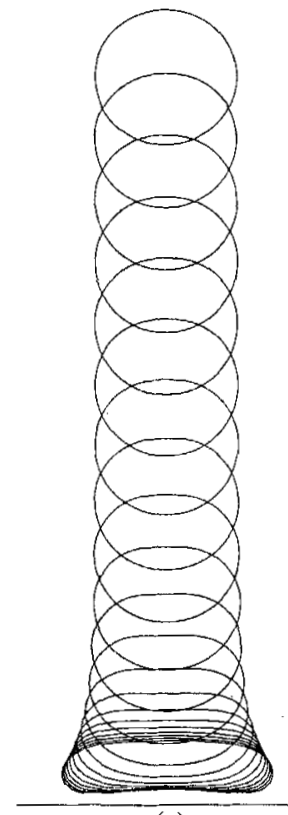

(c)

Figure $3(a-c)$. For eaption see facing page.

numerically determined film configurations at the timestep immediately prior to the formation of a dimple, are shown in figure 4 . These transition configurations all display a small, nearly flat region at the wall side of the drop near $\rho=0$. In each case, the dimple first forms in this flat region, at a relatively small radial distance from the $z$-axis. The apex of the dimple then moves radially outward.

The pertinent questions at this point are (i) what role do $C a$ and $\lambda$ play in determining the configurations shown in figure 4 , (ii) why does a dimple form once these configurations are established and (iii) what effect do $C a$ and $\lambda$ have once a dimple has formed?

Increasing $C a$ results in an overall increase in deformability of the drop throughout its entire range of motion. This is obvious from figure 3 . In addition, increasing capillary number results in 'onset' of dimpling farther from the wall. This statement may be made more precise by defining $Z_{\text {centre }}$, the $z$-axis location of the drop centre of mass and $Z_{\text {axis }}$, the distance along the $z$-axis from the plane to the nearest axis 


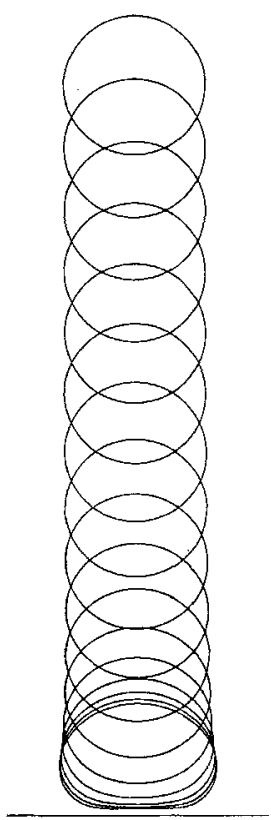

(d)

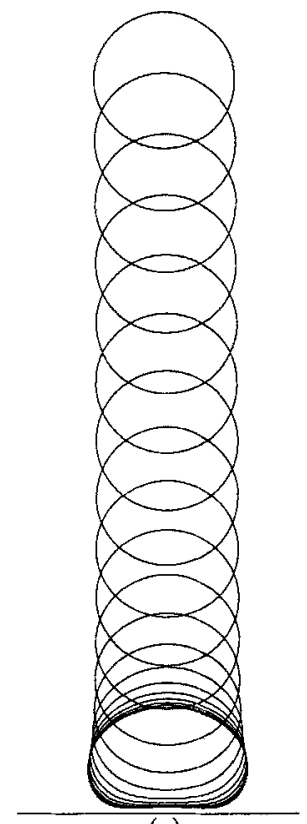

(g)

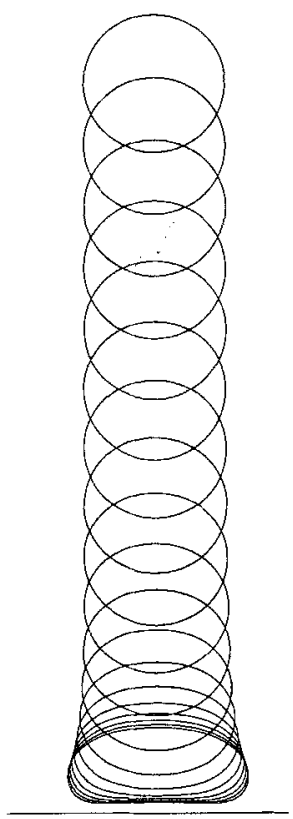

(e)

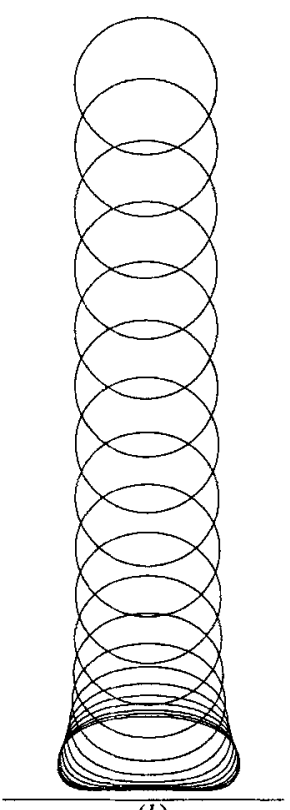

(h)
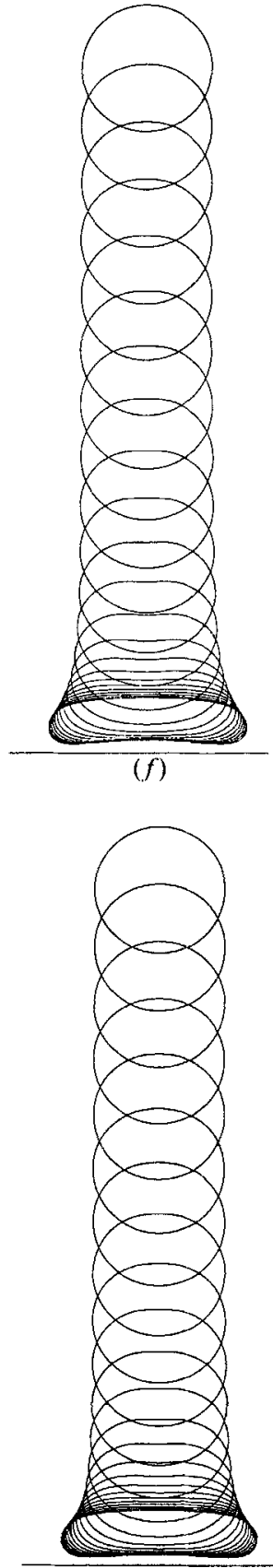

(i)

Figure 3. Evolution of drop shape. The dimensionless time between shape curves is 1. (a) $C a=$ $0.3, \lambda=0.3 ;(b) C a=1, \lambda=0.3 ;(c) C a=3, \lambda=0.3 ;(d) C a=0.3, \lambda=1 ;(e) C a=1, \lambda=1 ;(f)$ $C a=3, \lambda=1 ;(g) C a=0.3, \lambda=3 ;(h) C a=1, \lambda=3$; (i) $C a=3, \lambda=3$.

point of the drop (figure $1 b$ ). At the instant when dimpling is first numerically observed, $Z_{\text {axis }}$ and $Z_{\text {centre }}$ are largest for $C a=3$. The $Z_{\text {centre }}$ and $Z_{\text {axis }}$ values when dimpling is first observed both decrease with decreasing $C a$. This is clearly seen in figure 4 for the configuration immediately prior to dimpling. 


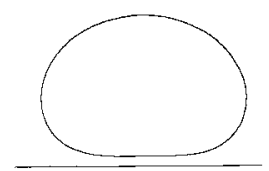

$\lambda=0.3$

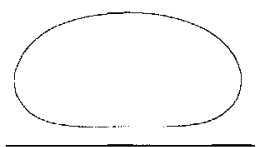

$\lambda=0.3$

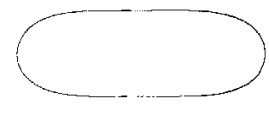

$\lambda=0.3$

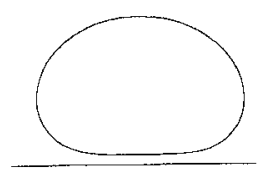

$\lambda=1$

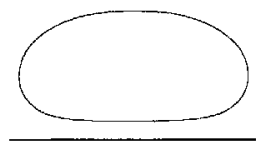

$\lambda=1$

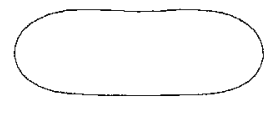

$\lambda=1$

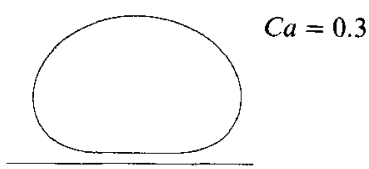

$\lambda=3$

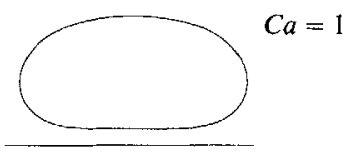

$\lambda=3$

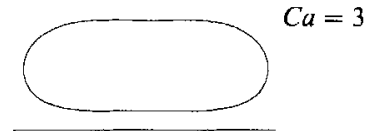

$\lambda=3$

Figure 4 . Drop configurations at the timestep immediately prior to dimpling.

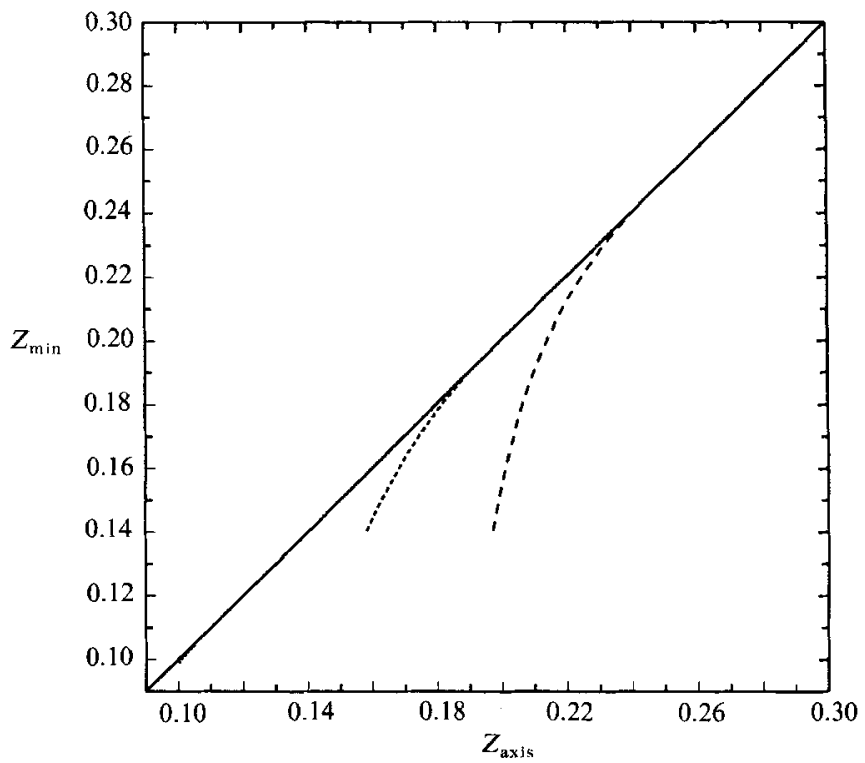

FiguRe 5. $Z_{\min }$ vs. $Z_{\text {axis }}$ for $\lambda$ fixed at 1 and $C a$ varying. Solid line corresponds to $Z_{\min }=Z_{\text {axis }}$ (i.e. no dimpling). $\cdots \cdots, C a=0.3 ;---, C a=1 ;-\cdots, C a=3$.

The depth of the dimpled region, and the location of the 'outer edge' of the dimple are best discussed in terms of $Z_{\min }$ and $\rho_{\min }$ (figure $1 b$ ). $Z_{\min }$ denotes the minimum distance along the $z$-direction between the drop and the plane wall, while $\rho_{\min }$ denotes the radial distance in cylindrical coordinates to the position on the drop at which $Z_{\min }$ occurs. Once a dimpled configuration has formed, for a given $Z_{\text {axis }}$, the depth of the dimple $Z_{\text {axis }}-Z_{\min }$ increases with increasing $C a$. In particular, for $C a=$ 0.3 dimpling is barely noticeable at the point where the calculation was terminated. 


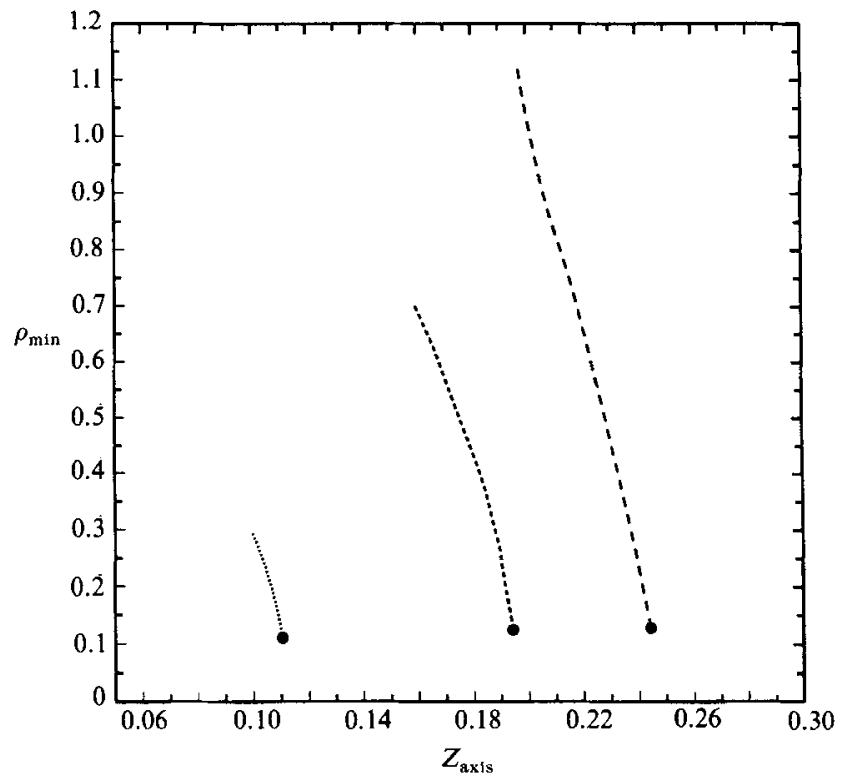

FiguRe 6. $\rho_{\min }$ vs. $Z_{\mathrm{ax} 1 \mathrm{~s}}$ for $\lambda$ fixed at 1 and $C a$ varying. $\cdots, C a=0.3 ;---, C a=1 ;---$, $C a=3$.

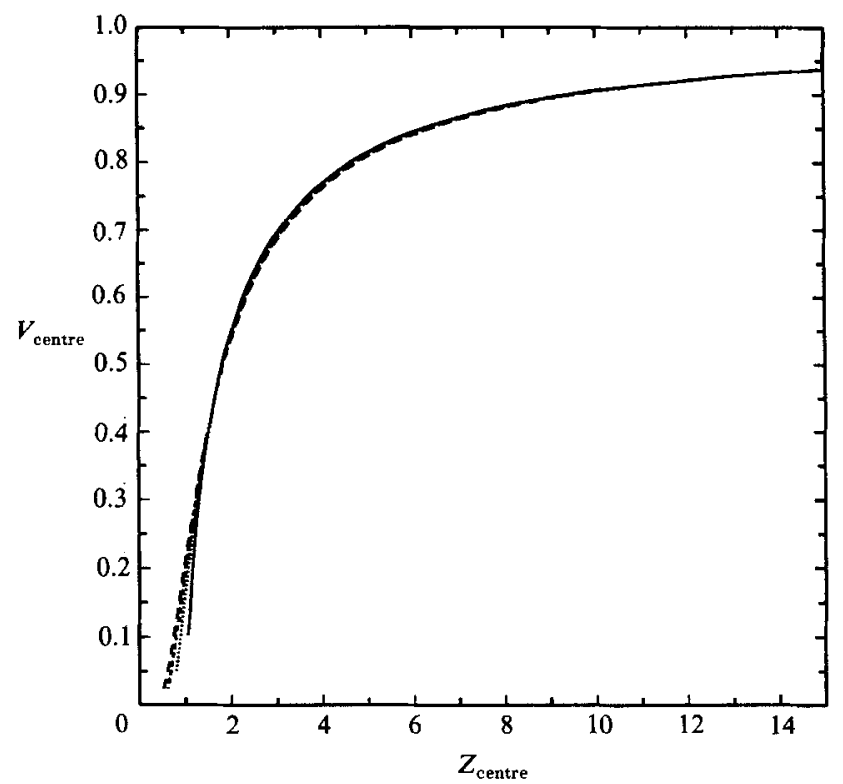

Figure 7. $V_{\text {centre }} v s . Z_{\text {centre }}$ for $\lambda$ fixed at 1 and $C a$ varying. $\cdots, C a=0.3 ; \cdots, C a=1 ;-,-C a$ $=3$. Solid line corresponds to the results of Bart (1968) for a spherical drop ( $C a=\infty$ limit).

However, as $C a$ increased to 1 and 3, the depth and width of the dimple region increased dramatically. This effect is illustrated in figures 5 and 6 for the case $\lambda=1$.

Increasing $\mathrm{Ca}$ also produces an increase in the deviation from sphericity at the side of the drop farthest from the wall. In fact, for $\mathrm{Ca}=3.0$, flattening and eventual indentation of the side of the drop farthest from the wall was observed. 


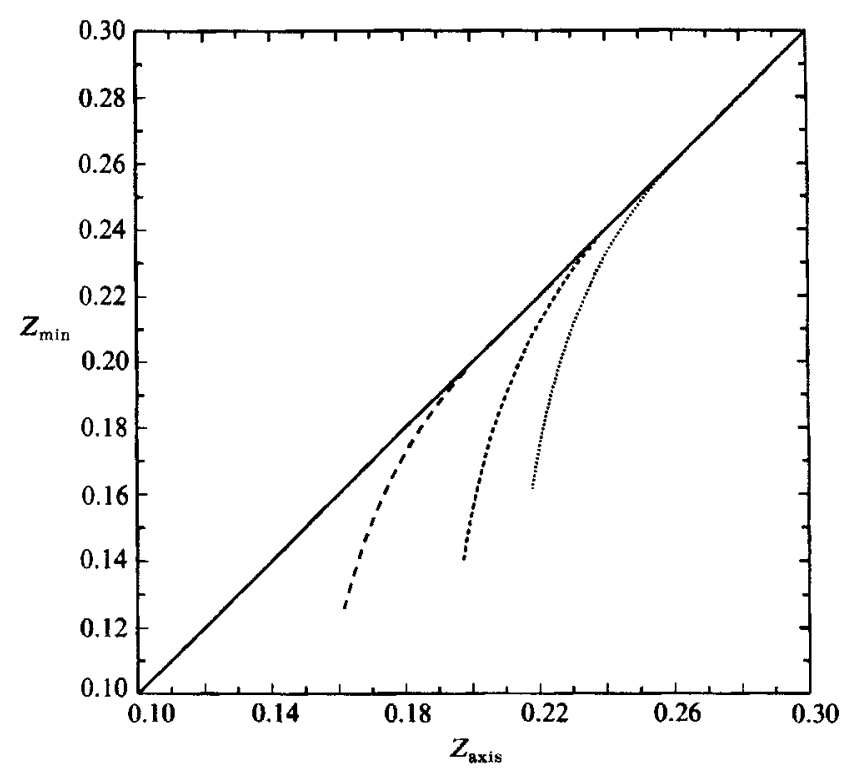

Fraver 8. $Z_{\min }$ vs. $Z_{\mathrm{axis}}$ for $C a$ fixed at 3 and $\lambda$ varying. Solid line corresponds to $Z_{\min }=Z_{\text {axls }}$ (i.e. no dimpling). $\cdots \cdots, \lambda=0.3 ; \cdots, \lambda=1 ;-\cdots, \lambda=3$.

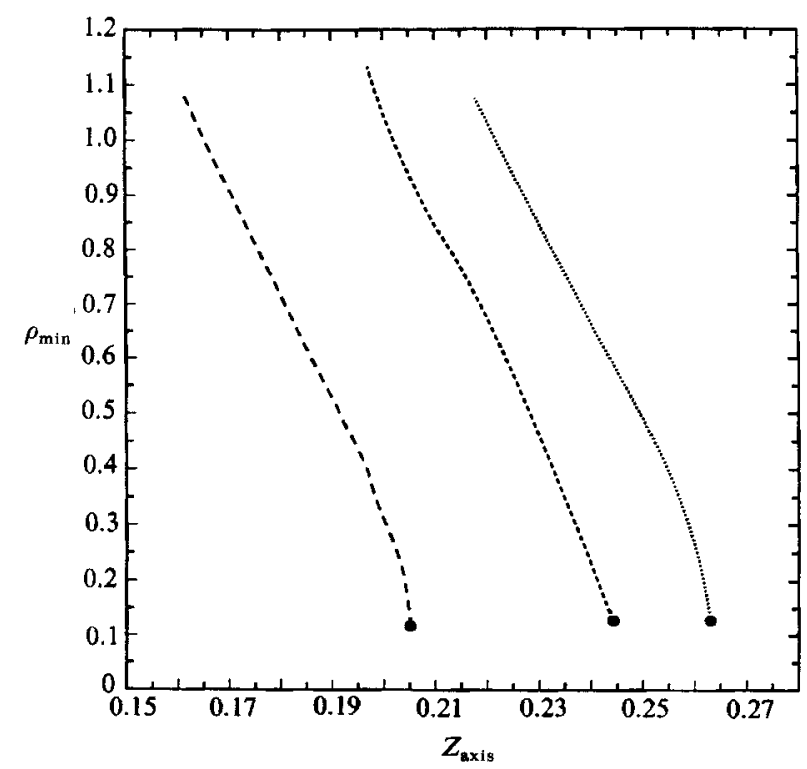

FIQURE 9. $\rho_{\min } v s . Z_{\mathrm{axis}}$ for $C a$ fixed at 3 and $\lambda$ varying. $\cdots, \cdot, \lambda=0.3 ; \cdots, \lambda=1 ;---, \lambda=3$.

The effect of $\mathrm{Ca}$ on the normalized centre of mass velocity is much less than might be anticipated from the large effect that $\mathrm{Ca}$ has on the drop shape. $V_{\text {centre }}$ plotted versus $Z_{\text {centre }}$ is shown in figure 7 for the case $\lambda=1$. Deviation from the nondeforming spherical results (solid line) is small. Far from the wall, these deviations are due to blunting of the wall side of the drop (and subsequent shift of the drop centre of mass away from the wall). Near the wall, deformation allows a mechanism of motion which the spherical drop is not allowed.

Conversely, what is the effect of varying $\lambda$ for fixed $\mathrm{Ca}$ ? Calculated shape details 


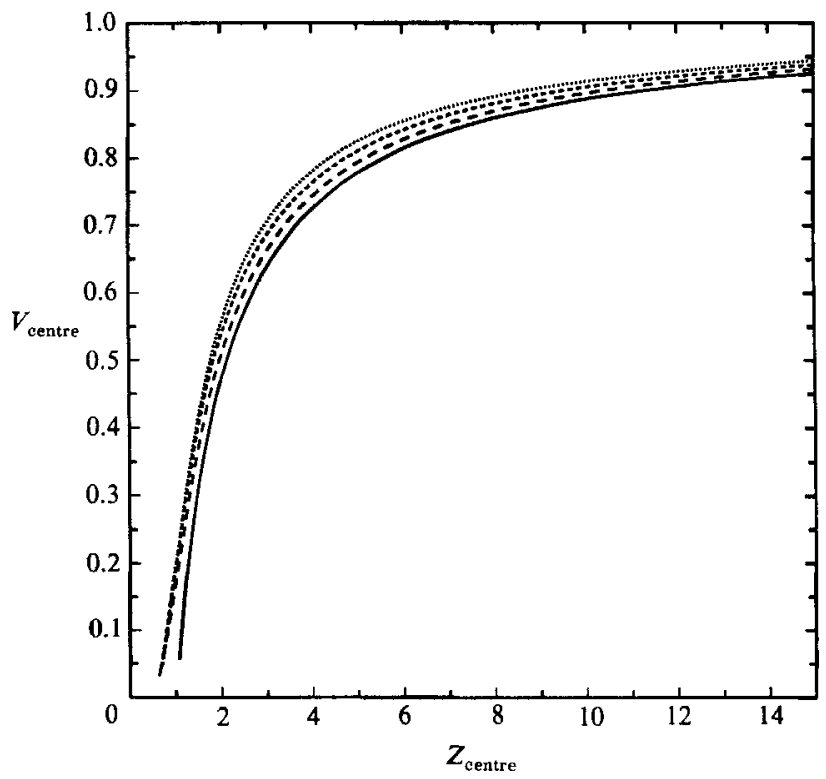

Fiaure 10. $V_{\text {oentre }}$ vs. $Z_{\text {centre }}$ for $C a$ fixed at 1 and $\lambda$ varying. $\cdots \cdots, \lambda=0.3 ;---, \lambda=1 ;---$, $\lambda=3$. Solid line corresponds to the results of Brenner (1961) for a solid sphere $(\lambda=\infty$ limit).

are remarkably insensitive to $\lambda$. For $C a$ fixed at 0.3 and $\lambda$ varying, figures $3 a, 3 d$ and $3 g$ are nearly indistinguishable as are the corresponding configurations shown in figure 4. For $C a$ fixed at 3 and 1, very slight configurational differences are observed. In particular, figure 4 demonstrates that increasing $\lambda$ corresponds to slightly smaller values of $Z_{\text {axis }}$ and $Z_{\text {centre }}$ immediately prior to dimpling. Even after dimpling has occurred, the effect of varying $\lambda$ in the $C a=1$ and 3 cases is relatively small. The general trends in the dimpled configuration are shown in figures 8 and 9 . The $\rho_{\min }$ value where the dimple is first formed is insensitive to $\lambda$ (figure 9 , where the dark circle denotes this value). The shapes of the curves in figures 8 and 9 are remarkably similar for different $\lambda$. In short, the 'dynamics' are roughly the same for each $\lambda$. Once again, the value of $\lambda$ determines the starting $Z_{\text {axis }}$ values where the curves begin.

Variation in $\lambda$ does have a significant effect on the normalized centre of mass velocity. This is demonstrated in figure 10 . At a given $Z_{\text {centre location, drops with }}$ higher viscosity relative to the suspending fluid show a larger wall interaction (corresponding to lower $V_{\text {centre}}$ ). This effect diminishes significantly in the latest stages of deformation when the drop is closest to the wall. Thus, the major effect of $\lambda$ is to set the relative rate at which the drop evolves, up to the point of dimpling.

Thus far, the gross geometrical details of deformation and the relative rate at which this deformation takes place have been correlated with $C a$ and $\lambda$. A careful examination of the velocity and modified pressure fields provides additional insight into the dynamics of dimple formation.

Equations (10) allow computation of velocity and pressure fields in the two fluid domains. Figure 11(a) shows the velocity field obtained for $C a=1, \lambda=1$ in a dimpled configuration. Here the velocity is calculated relative to a fixed wall. Figure $11(b)$ shows the analogous velocity field, with velocity measured relative to the centre of mass of the drop. Figures 12 and $13(a, b)$ show the velocity fields for the dimpled configurations with parameters $C a=0.3, \lambda=1$ and $C a=3, \lambda=1$, respectively. These figures indicate that, once a dimple is formed, the largest relative 

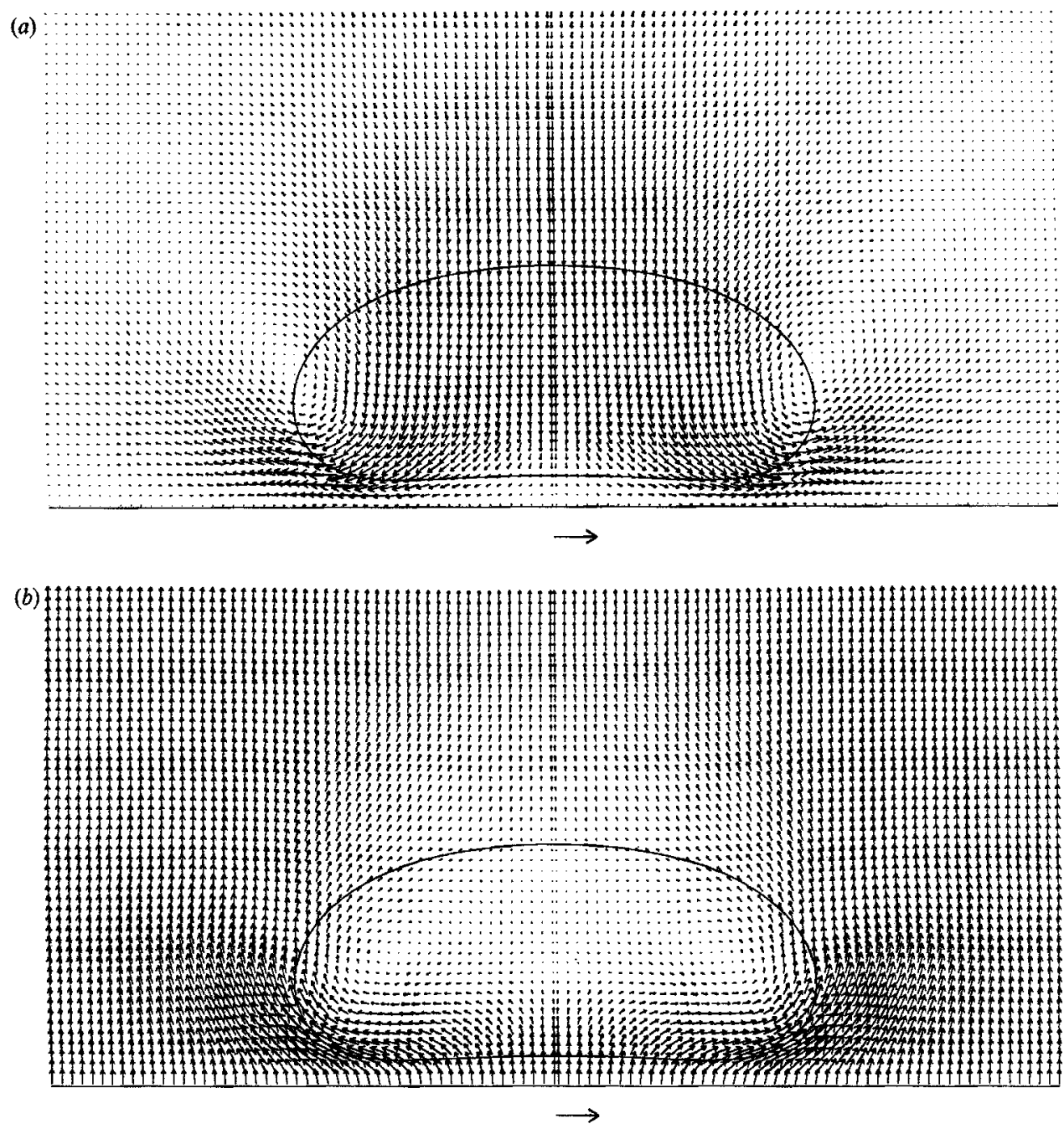

Figure 11 (a) Velocity field for $C a=1, \lambda=1, Z_{\text {centre }}=0.615$. The horizontal arrow below the wall corresponds in length to $\frac{1}{10} U$ and thus indicates scale. Velocities are measured relative to an origin fixed on the wall. $(b)$ As $(a)$, except velocities are measured relative to an origin fixed at the centre of mass of the drop.

motion occurs at the outer edge of the dimple. In particular, the downward motion of the film at $\rho=0$ is small in comparison to the downward motion of the outer edge of the dimpled region. In short, the dimples increase in depth $\left(Z_{\text {axis }}-Z_{\text {min }}\right)$ with time.

It is instructive to focus on the velocity fields in the film region immediately prior to the dimple formation. Figures 14 and 15 show the radial and $z$-components of the velocity in the film for $C a=1$ and $\lambda=1$. At the next numerical timestep a dimple has formed with its apex at the radial position marked by the vertical line at the wall. From these plots it is easily seen how a dimple will form in terms of velocity field variation. Near $\rho=0$ the face of the drop nearest the wall is approximately flat. The downward component of velocity increases as one moves radially outward. Although this increase in downward velocity is small, near $\rho=0$ the surface is nearly flat and thus only a slight increase in downward velocity is sufficient to form the dimple. 


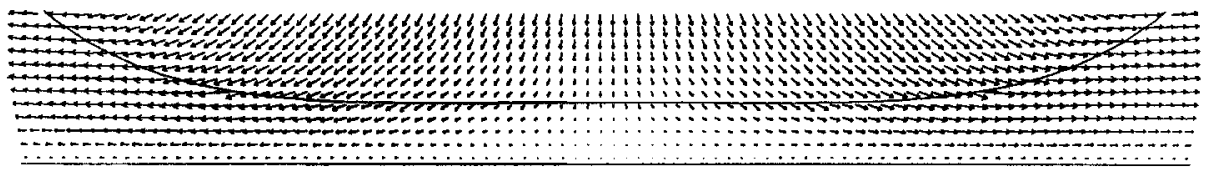

Figure 12. Velocity field for $C a=0.3, \lambda=1, Z_{\text {centre }}=0.787$. The horizontal arrow below the wall corresponds in length to $\frac{1}{10} U$ and thus indicates scale.

(a)

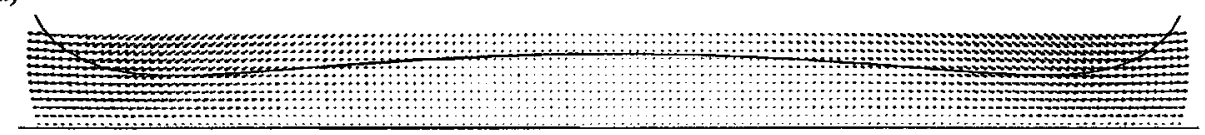

(b)

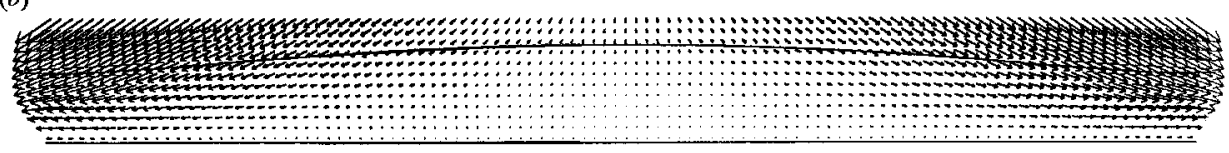

Figure 13. Velocity field for $C a=3, \lambda=1, Z_{\text {centre }}=0.477$. (a) The entire film. The horizontal arrow below the wall corresponds in length to $\frac{1}{10} U$ and thus indicates scale. $(b)$ The central region of the film. The horizontal arrow below the wall corresponds in length to $\frac{1}{100} U$ and thus indicates scale.

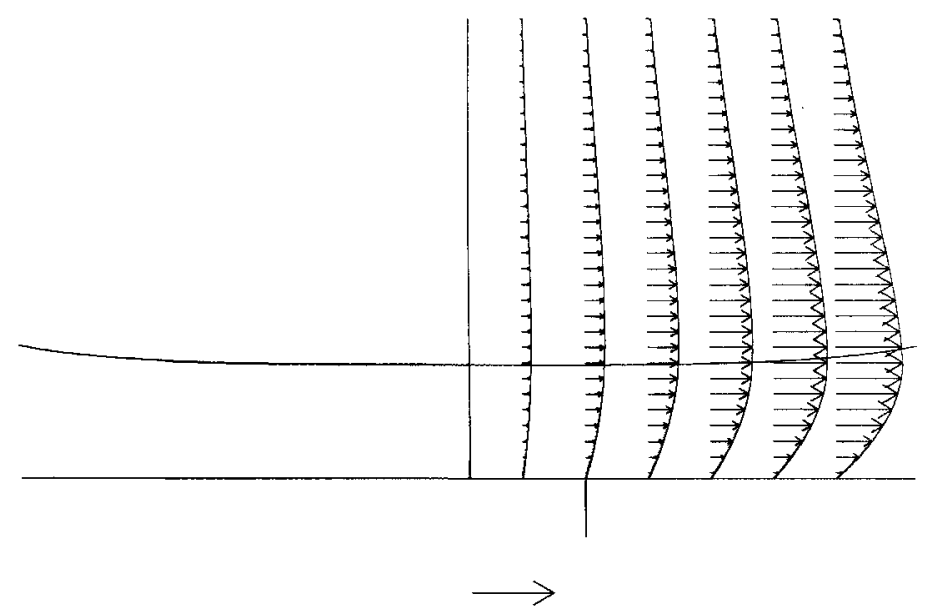

FrauRE 14. The $\rho$-component of velocity for $C a=1, \lambda=1, Z_{\text {centre }}=0.757$. The next numerical timestep corresponds to the first formation of a dimple. The vertical line below the wall corresponds to the radial location at which the dimple initially forms. The horizontal arrow below the wall corresponds in length to $\frac{1}{10} U$ and thus indicates scale.

Stokes equations are physically a balance between the forces due to modified pressure and the forces due to viscous stresses. Viscous stresses correspond to velocity variation. We have examined the velocity fields. The next step is to consider the modified pressure fields.

Film-drainage theory employing the lubrication approximation emphasizes the 

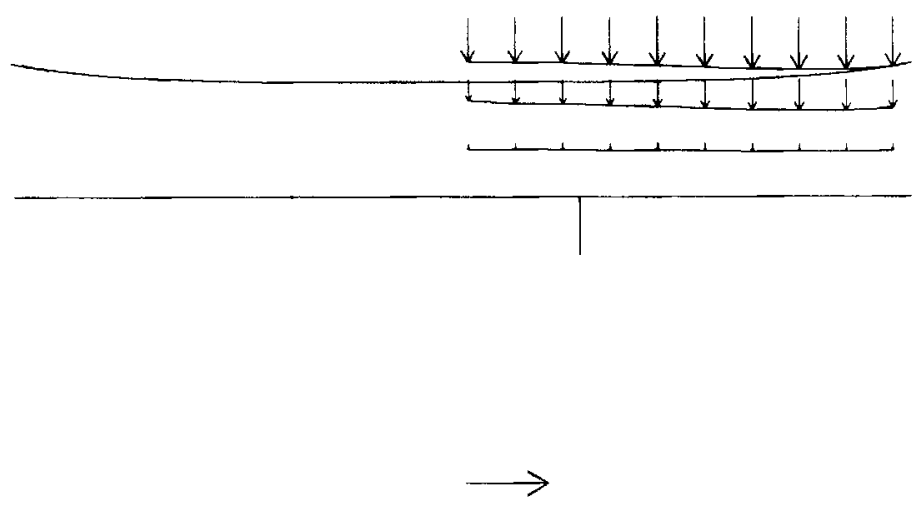

FigURE 15 . The $z$-component of velocity for $C a=1, \lambda=1, Z_{\text {centre }}=0.757$. The next numerical timestep corresponds to the first formation of a dimple. The vertical line below the wall corresponds to the radial location at which the dimple initially forms. The horizontal arrow below the wall corresponds in length to $\frac{1}{10} U$ and thus indicates scale.

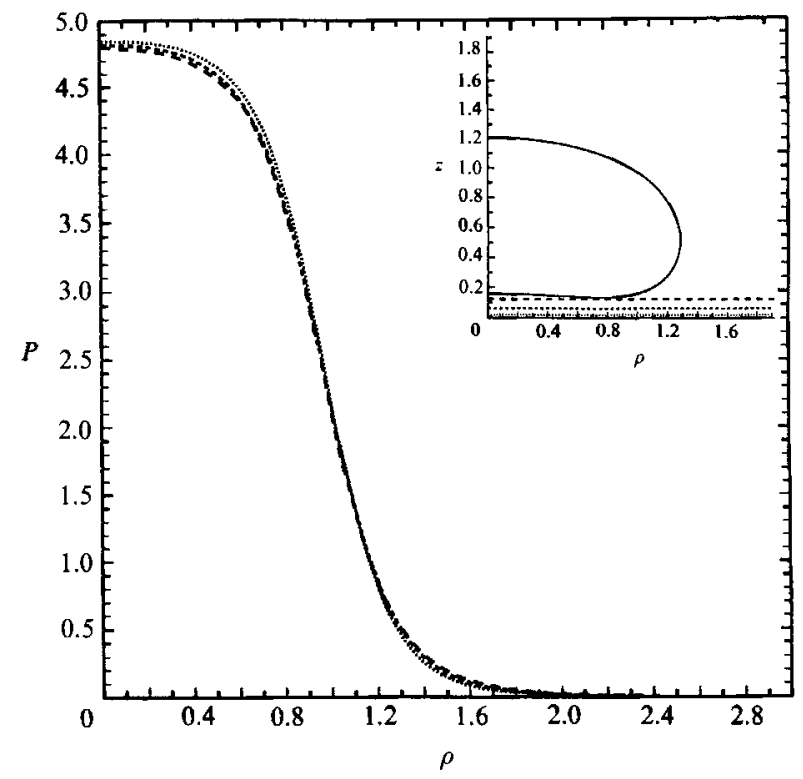

Figure 16. Pressure for $C a=1, \lambda=1$, and $Z_{\text {centre }}=0.615$ plotted against radial distance. $\cdots \cdots$, $z=0.01 ;-\cdots--, z=0.06 ;---, z=0.12$. Inset: The corresponding shape and $z$-values.

importance of variation in pressure with radial position. Variation of pressure with $z$ is neglected. The velocities calculated at the surface of the film are directly related to the assumed radial variation in pressure. As a consequence, the evolution of the film shape is critically dependent on the assumed variation in pressure. What do the numerically calculated pressure fields look like after a dimple has formed? Figures 16-18 show calculated modified pressure fields corresponding to the configurations of figures 11-13. The most obvious variation in pressure occurs at the outer edge of the film region. Here $p_{2}$ rapidly decreases radially outward. Equivalently, $\partial p_{2} / \partial \rho$ is negative and large in absolute magnitude. In each case, $p_{2}$ in the film region decreases with $z$ at a fixed $\rho$ location. In other words, $\partial p_{2} / \partial z$ is negative in the film region. For the significantly dimpled case of $C a=3, \partial p_{2} / \partial \rho$ is positive from $\rho=0$ outward to the 


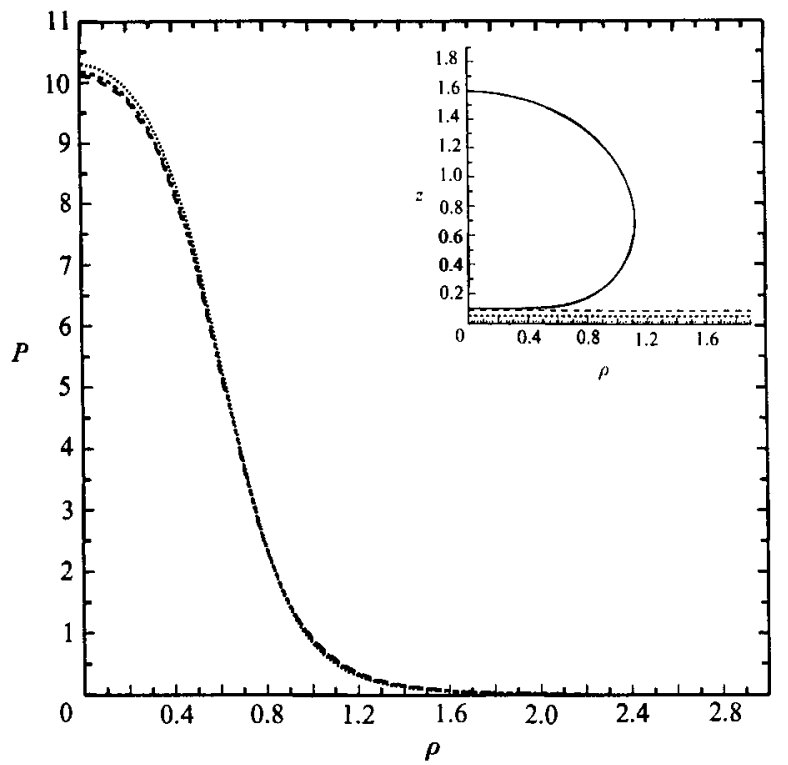

Figure 17. Pressure for $C a=0.3, \lambda=1$, and $Z_{\text {centre }}=0.787$ plotted against radial distance. $\cdots \cdots$, $z=0.01 ;----, z=0.05 ;---, z=0.087$. Inset: The corresponding shape and $z$-values.

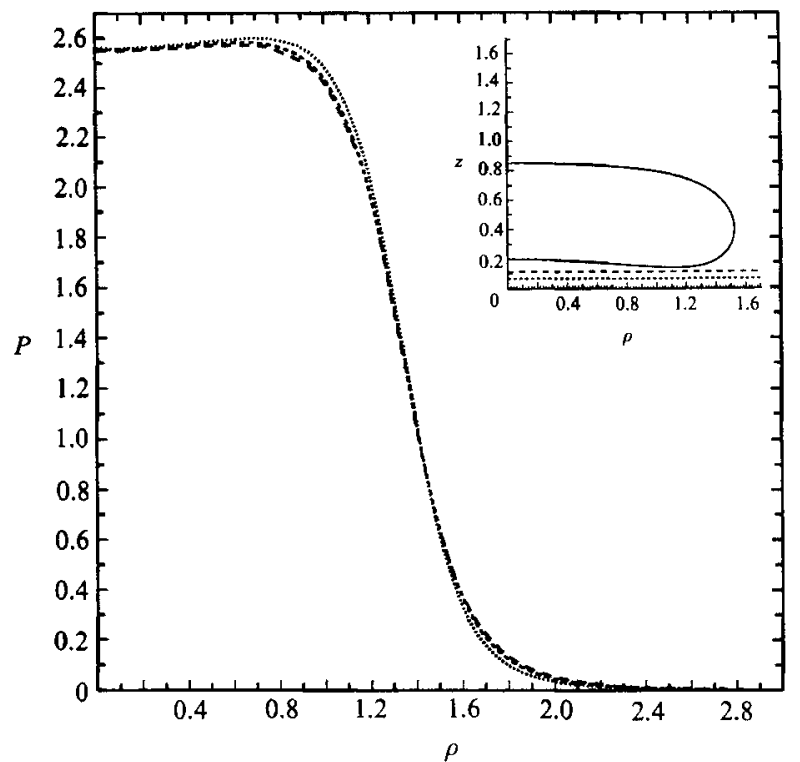

Figure 18. Pressure for $C a=3, \lambda=1$, and $Z_{\text {centre }}=0.477$ plotted against radial distance. $\cdots \cdots$, $z=0.01 ;---, z=0.065 ;-\cdots-, z=0.11$. Inset: The corresponding shape and $z$-values.

edge of the dimple and negative beyond the dimple. On the other hand, for the $C a=1$ and $C a=0.3$ cases, where the dimple is in its infancy compared to the $C a=3$ case, $\partial p_{2} / \partial \rho$ is negative throughout the film region. For $C a=3, \partial p_{2} / \partial \rho$ and $\partial p_{2} / \partial z$ are extremely small in the nearly stagnant central flow region. For the other cases $\partial p_{2} / \partial \rho$ and $\partial p_{2} / \partial z$ are roughly of the same order of magnitude near $\rho=0 . \partial p_{2} / \partial \rho$ grows rapidly more negative near the edge of the film region, while here $\partial p_{2} / \partial z$ is small in magnitude by comparison. 


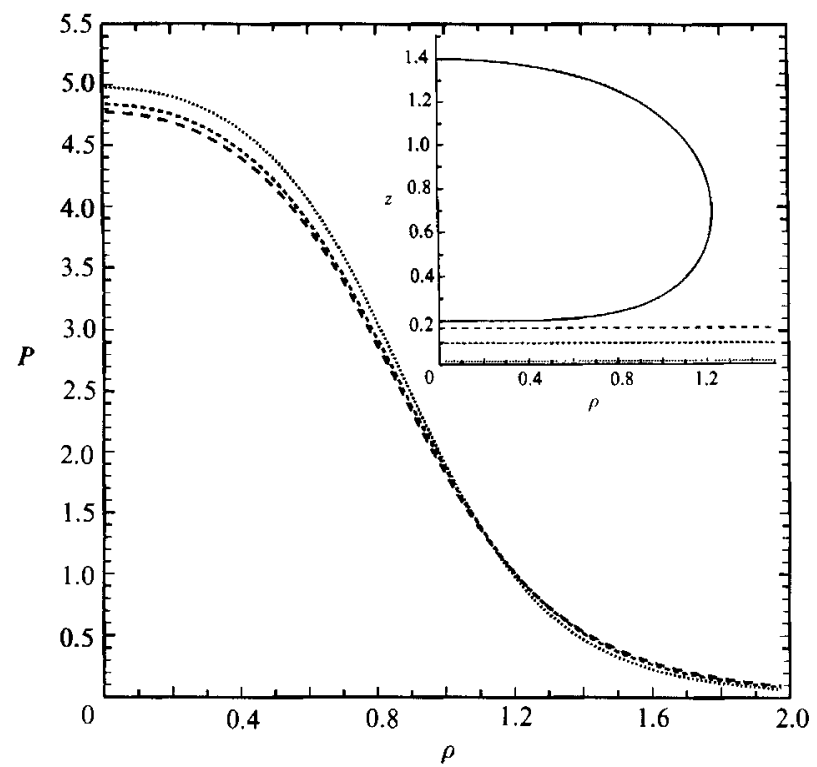

Figure 19. Pressure for $C a=1, \lambda=1$, and $Z_{\text {centre }}=0.757$ plotted against radial distance. The next numerical timestep corresponds to the first formation of a dimple at $\rho=0.125 . \cdots \cdots, z=0.01$; $\cdots-, z=0.1 ;-\cdots, z=0.17$. Inset: The corresponding shape and $z$-values.

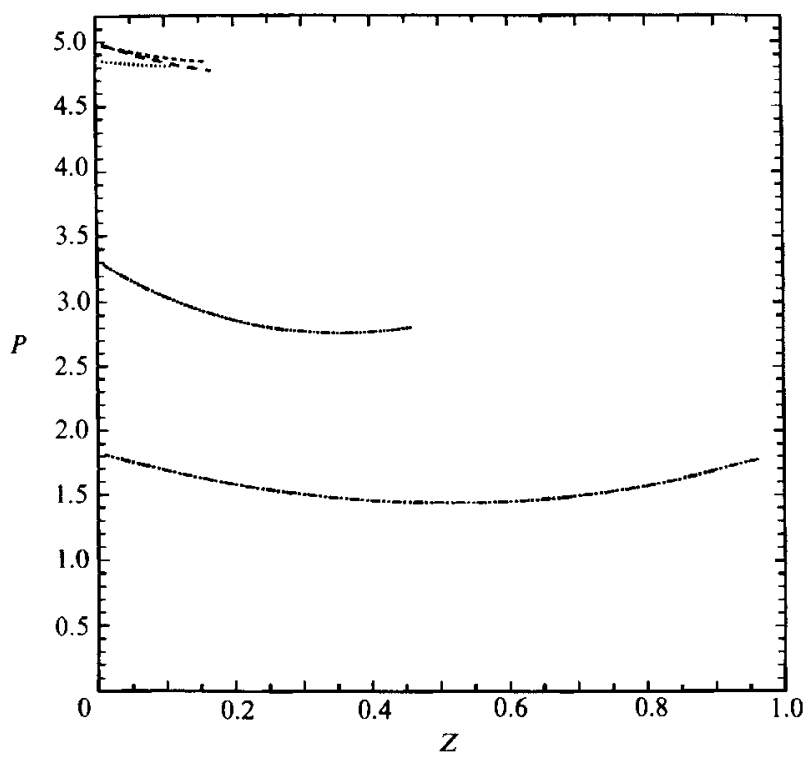

Figure 20. Pressure for $C a=1, \lambda=1$, and $\rho=0.01$ plotted against vertical distance. Each curve corresponds to a different $Z_{\text {centre }}$ location. $\cdots \cdots, Z_{\text {centre }}=0.615\left(Z_{\text {axis }}=0.1549\right) ;----, Z_{\text {centre }}=$ $0.6918\left(Z_{\text {axis }}=0.1756\right) ;--, Z_{\text {centre }}=0.757\left(Z_{\text {axis }}=0.1970\right) ;-\cdots-, Z_{\text {centre }}=1.320\left(Z_{\text {axis }}=0.5221\right)$; $-\cdots, Z_{\text {centre }}=1.192\left(Z_{\text {axis }}=1.028\right)$.

What do the pressure fields look like, immediately prior to dimple formation? Figure 19 shows the modified pressure for the $C a=1, \lambda=1$ case immediately prior to dimple formation. The largest pressure variation is at the edge of the drop, where pressure rapidly decreases radially outward. In contrast, for this configuration, the 


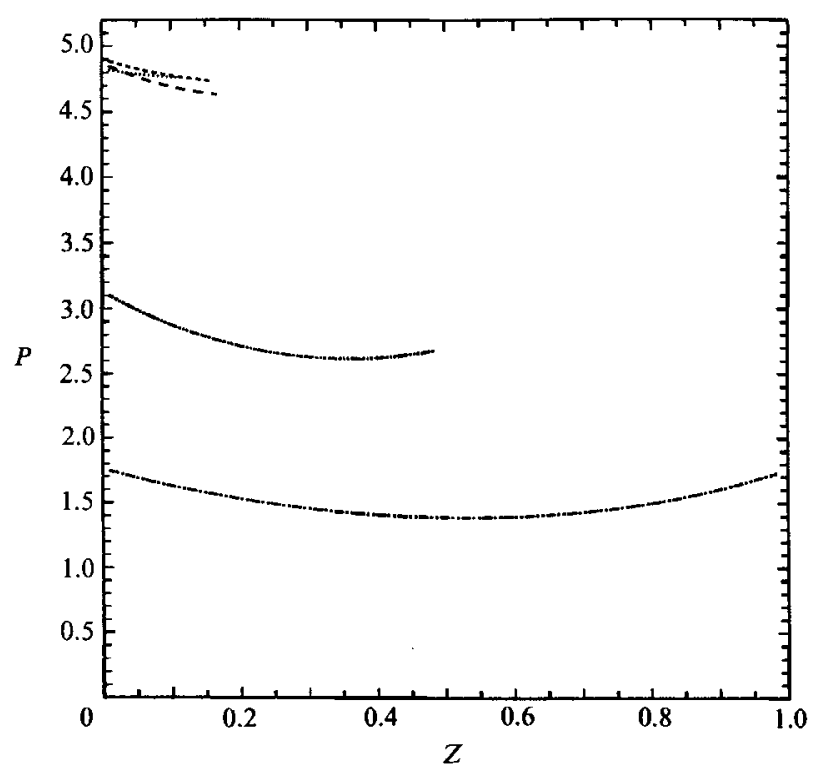

Figure 21. Pressure for $C a=1, \lambda=1$, and $\rho=0.26$ plotted against vertical distance. Each curve corresponds to a different $Z_{\text {centre }}$ location. $\cdots \cdots, Z_{\text {centre }}=0.615\left(Z_{\text {axis }}=0.1549\right) ;---, Z_{\text {centre }}=$ $0.6918\left(Z_{\text {axis }}=0.1756\right) ;--,, Z_{\text {centre }}=0.757\left(Z_{\text {axis }}=0.1970\right) ;-\cdots-, Z_{\text {centre }}=1.320\left(Z_{\text {axis }}=0.5221\right)$; $\cdots-, Z_{\text {centre }}=1.192\left(Z_{\mathrm{axis}}=1.028\right)$.

dimple first forms at $\rho=0.125$ where the radial pressure variation is small relative to pressure variation with $z$. This is at odds with thin-film lubrication assumptions.

How does the pressure field vary as the drop approaches the wall ? In particular, how does $\partial p_{2} / \partial z$ vary near the centre of the drop, as the drop approaches the wall ? The evolution of pressure fields as the $C a=1, \lambda=1$ drop approaches the wall is shown in figures 20-22. For each plot, a radial distance is fixed and pressure is calculated for $z$-values beginning at the wall and up to the drop surface. Each curve in the figures then corresponds to a different $Z_{\text {centre }}$ location. These figures illustrate the fact that for small $\rho$ and large $Z_{\text {centre }}, \partial p_{2} / \partial z$ is positive near the drop surface. As $Z_{\text {centre }}$ decreases (i.e. the drop moves toward the wall) $\partial p_{2} / \partial z$ becomes negative over the entire film height. As the drop moves closer to the wall $\partial p_{2} / \partial z$ eventually decreases in magnitude.

Summarizing, pressure variation with $z$ may be negligible in the latest stages of dimple formation. In contrast, when the dimple first forms, pressure variation with $z$ is not small relative to pressure variation with $\rho$.

The significance of a negative $\partial p_{2} / \partial z$, at the onset of dimpling may be seen from an elementary analysis of Stokes equations.

Writing system (1) in pressure-vorticity form (see Happel \& Brenner $1965 § 3.1$; below velocities and pressures refer to fluid 2 ):

$$
\begin{aligned}
& \frac{\partial p}{\partial \rho}=\frac{\partial}{\partial z}\left[-\frac{\partial v_{z}}{\partial \rho}+\frac{\partial v_{\rho}}{\partial z}\right] \\
& \frac{\partial p}{\partial z}=\frac{1}{\rho} \frac{\partial}{\partial \rho}\left(\rho\left[\frac{\partial v_{z}}{\partial \rho}-\frac{\partial v_{\rho}}{\partial z}\right]\right) .
\end{aligned}
$$




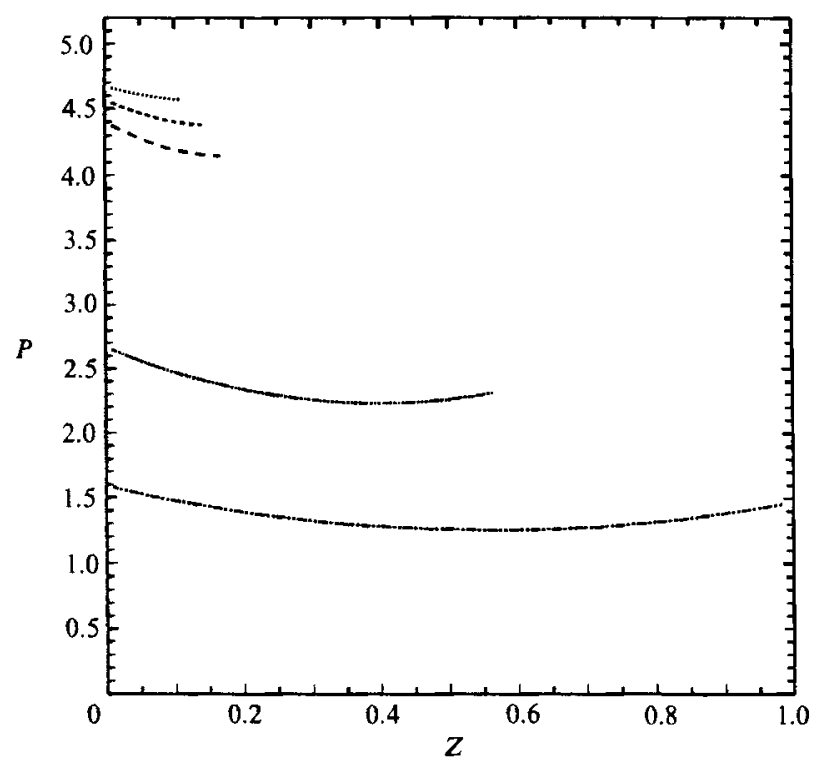

Figure 22. Pressure for $C a=1, \lambda=1$, and $\rho=0.5$ plotted against vertical distance. Each curve corresponds to a different $Z_{\text {centre }}$ location. $\cdots \cdots, Z_{\text {centre }}=0.615\left(Z_{\text {axis }}=0.1549\right) ; \cdots--, Z_{\text {centre }}=$ $0.6918\left(Z_{\text {axis }}=0.1756\right) ;---, Z_{\text {centre }}=0.757\left(Z_{\text {axis }}=0.1970\right) ;-\cdots-, Z_{\text {centre }}=1.320\left(Z_{\text {axis }}=0.5221\right)$; $-\cdots-, Z_{\text {centre }}=1.192\left(Z_{\text {axls }}=1.028\right)$.

Integrating these equations from 0 to $\rho$ and from $z_{0}$ to $z$ gives

$$
\begin{aligned}
& -\frac{\partial v_{z}}{\partial \rho}+\frac{\partial v_{\rho}}{\partial z}=\int_{z_{0}}^{z} \frac{\partial p}{\partial \rho} \mathrm{d} z+F(\rho), \\
& -\frac{\partial v_{z}}{\partial \rho}+\frac{\partial v_{\rho}}{\partial z}=-\frac{1}{\rho} \int_{0}^{\rho} \rho \frac{\partial p}{\partial z} \mathrm{~d} \rho,
\end{aligned}
$$

where finiteness at $\rho=0$ has been used. Differentiating (20) with respect to $z$ and applying the differential operator $(1 / \rho)(\partial / \partial \rho) \rho(\quad)$ to $(19)$, and using the harmonicity of pressure gives

$$
\frac{1}{\rho} \frac{\partial}{\partial \rho}(\rho F(\rho))=\left.\frac{\partial p}{\partial z}\right|_{z=z_{0}}
$$

Solving (once again assuming finiteness at $\rho=0$ ) yields

$$
-\frac{\partial v_{z}}{\partial \rho}+\frac{\partial v_{\rho}}{\partial z}=-\frac{1}{\rho} \int_{0}^{\rho} \rho \frac{\partial p}{\partial z} \mathrm{~d} \rho=\int_{z_{0}}^{z} \frac{\partial p}{\partial \rho} \mathrm{d} z-\left.\frac{1}{\rho} \int_{0}^{\rho} \rho \frac{\partial p}{\partial z}\right|_{z=z_{0}} \mathrm{~d} \rho
$$

This equation applies in any rectangle $[0, \rho] \times\left[z_{0}, z\right]$ contained in fluid 2 . In particular, since the film depicted in figures 14 and 15 is nearly flat, apply this equation on a disk, $z=z_{0}, \rho \in\left[0, \rho_{f}\right]$ parallel to the film where $z_{0}$ lies just below the upper surface of the film and $\rho_{f}$ is the radial location where dimpling first occurs:

$$
\left.\frac{\partial\left(-v_{z}\right)}{\partial \rho}\right|_{z=z_{0}, \rho-\rho_{f}}=-\left.\frac{\partial v_{\rho}}{\partial z}\right|_{z=z_{0}, \rho-\rho_{f}}-\left.\frac{1}{\rho_{f}} \int_{0}^{\rho_{f}} \rho \frac{\partial p}{\partial z}\right|_{z=z_{0}} \mathrm{~d} \rho .
$$

Figure 14 indicates that $\left.\left(\partial v_{\rho} / \partial z\right)\right|_{z=z_{0}, \rho-\rho_{f}}$ will be nearly zero or small and positive. 
Dimpling at $\rho_{f}$ can only occur in this configuration if $\left.\left(\partial\left(-v_{z}\right) / \partial \rho\right)\right|_{z=z_{0}, \rho=\rho_{f}}$ is positive. Assuming that $z_{0}$ is sufficiently close to the film surface that continuity of velocity will ensure that what happens at $\left(\rho_{f}, z_{0}\right)$ is representative of what happens at the film surface, $\left(\rho_{f}, z_{\mathrm{film}}\right)$, then it is necessary that $\left.(\partial p / \partial z)\right|_{z=z_{0}}$ be negative in a region near the drop surface. This analysis demonstrates that the Stokes equations in fluid 2 when applied to a flat-film configuration only allow dimple formation if $\partial p / \partial z$ is negative near the drop surface. Let us suppose, in contrast, that lubrication theory is applied (e.g. Hartland 1969; Lin \& Slattery 1982, where gravity is neglected or Ascoli 1988, where Hartland's analysis is extended to include gravity) when the film is locally flat. The result, for a locally flat configuration is that no local thinning of the interface occurs. $\dagger$ This is a direct consequence of neglect of pressure variation with $z$ in the lubrication theory!

\subsection{Discussion}

The assumptions relevant to film-drainage theory listed in the introduction may now be assessed.

def

(i) Films have been assumed thin in the sense that $\epsilon=h / R \ll 1$. This assumption has been used to neglect various terms in the equations of motion in the context of 'lubrication' or boundary-layer approximations. It is anticipated that the simplifying approximations made in these theories are increasingly accurate as $\epsilon \rightarrow 0$. Taking $h$ to be $Z_{\text {axis }}$ at the instant the dimple forms and $R$ to be the undeformed radius of the drop (for the numerical cases shown, this choice of $R$ provides a reasonable measure of the radial extent of the film region), at the onset of dimpling, the current numerics predict $\epsilon$ in the range $0.1-0.3$. These values of $\epsilon$ are by no means vanishingly small which suggests that the lubrication approximations may not be accurate at the onset of dimple formation.

(ii) Jones and Wilson (1978) applied thin-film arguments to the related problem of a drop approaching a deformable interface. They considered the film to be composed of a dimple region and a central film region. If $u_{t}$ denotes tangential velocity, the tangential stress in the central film is of the order of $\mu_{2} u_{t} / h$ while the stress in the drop is of the order $\mu_{1} u_{t} / R$. Viscosity ratio, $\lambda$, is assumed to be $O(1)$ while $\epsilon=h / R$ is assumed small. Continuity of velocities indicates $u_{t}$ in the central film and in the drop are of the same order. The conclusion Jones \& Wilson reach is that the tangential stresses cannot match to leading order and thus $\partial u_{t} / \partial n=0$. The viscous contribution within the drop to the tangential stress does not come into play. In the dimple region this analysis breaks down. Here the appropriate length scaling in the drop is $h$ (the order of size of the dimple) and the tangential stress in the drop is of the same order as the film tangential stress. Thus Jones \& Wilson conclude that viscous effects in the drop must be considered in the dimple region. Immediately prior to dimple formation, their argument indicates $\partial v_{\rho} / \partial z=0$ throughout the film region. Figure 14 indicates that $\partial v_{\rho} / \partial z$ is small at the interface in the central region of the film. However, this may be deceptive since in the initial stages of dimple formation (21) indicates that an accurate estimate for $\partial v_{\rho} / \partial z$ at the interface may be crucial. Thus, it is difficult to assess the error of this approximation clearly.

(iii) The dimensional equivalent of (5), when rescaled, provides the necessary information to determine when for a 'thin' film gravity effects may be neglected

+ In these lubrication analyses, if $g(\rho, t)$ represents the vertical displacement of the film from the wall at time $t$, then $\partial g(\rho, t) / \partial t$ is found to depend on $\partial g / \partial \rho$ and higher $\rho$ derivatives. Thus $\partial g / \partial t=$ 0 locally if the interface is locally flat. 
relative to surface tension effects. Scaling $\rho$ with $R, z$ with $h$ and curvature with $\epsilon / R$ leads to the parameter (Ascoli 1988)

$$
M=3\left(\frac{R}{a}\right)^{2}\left(\frac{1+\frac{3}{2} \lambda}{1+\lambda}\right) C a .
$$

Roughly speaking, $M$ is the ratio of gravitational effects to surface tension effects. $M \ll 1$ indicates gravity is negligible relative to surface tension. Only in the limit $C a \ll 1$ will $M$ be small. None of the numerical cases considered here approach this limit.

(iv) Drop deformation increases with increasing $\mathrm{Ca}$. Thus the assumption of spherical drop shape outside the film region is limited to $C a \ll 1$. Similarly, outside of the dimpled region, invariance of drop shape is limited to the small $\mathrm{Ca}$ regime.

Dimple evolution is observed to occur in several stages. Initially a configuration is established in which the interface is nearly flat at the central region of the film, and then gradually slopes upward at the edge of the film. In this configuration, $\partial p_{2} / \partial z$ is observed to be negative near $\rho=0$. A negative value of $\partial p_{2} / \partial z$ has been shown to be crucial to the observed increase in downward velocity as one moves radially outward across the locally flat film. It is this increase in downward velocity that forms the initial dimple. Lubrication theory neglects the critical variation of pressure with $z$. As a consequence, lubrication theory predicts no local deformation of a locally flat interface.

In the later stages of dimple growth (here observed for $C a=3$ ), velocity at the central region of the film is dramatically smaller than near the edge of the dimple. Here fluid is 'trapped' by the dimple and corresponding pressure fields indicate that pressure increases radially outward to the edge of the dimple, and then decreases to the stagnant fluid value. The quantity, $\partial p_{2} / \partial z$, is seen to decrease in magnitude in the later stages of dimple evolution. It is only for this last stage of dimple evolution that the lubrication assumption of negligible pressure variation with $z$ may be valid.

This work was supported, in part, by a grant from the Fluid Mechanics program of the National Science Foundation.

\section{REFERENCES}

AscolI, E. P. 1988 Low-Reynolds number effects of walls on solid particles, bubbles and drops. $\mathrm{PhD}$ thesis, California Institute of Technology.

Ascoli, E. P., Dandy, D. S. \& LeaL, L. G. 1989 Low-Reynolds number hydrodynamic interaction of a solid particle with a planar wall. Intl J. Numer. Meth. Fluids 9, 651-688.

BART, E. 1968 The slow unsteady settling of a fluid sphere toward a flat fluid interface. Chem. Engng Sci. 23, 193-210.

BLAKE, J. R. 1971 A note on the image system for a Stokeslet in a no-slip boundary. Proc. Camb. Phil. Soc. 70, 303-310.

Brevner, H. 1961 The slow motion of a sphere through a viscous fluid towards a plane surface. Chem. Engng Sci. 16, 242-251.

Cнг, B. K. 1986 The motion of immiscible drops and the stability of annular flow. PhD thesis, California Institute of Technology.

Chi, B. K. \& Leal, L. G. 1989 A theoretical study of the motion of a viscous drop toward a fluid interface at low Reynolds number. J. Fluid Mech. 201, 123-146.

Dimitrov, D. S. \& Ivanov, I. B. 1978 Hydrodynamics of thin liquid films. On the rate of thinning of microscopic films with deformable interfaces. J. Colloid Interface Sci. 64, 97-106.

Frankel, S. P. \& Mrseis, K. J. 1962 On the 'dimpling' during the approach of two interfaces. J. Phys. Chem. 66, 190-191. 
Geller, A. S., Lee, S. H. \& Leal, L. G. 1986 The creeping motion of a spherical particle normal to a deformable interface. J. Fluid Mech. 169, 27-69.

Haberman, W. L. \& Sayre, R. M. 1958 Motion of rigid and fluid spheres in stationary and moving liquids inside cylindrical tubes. David Taylor Model Basin Rep. No. 1143, Washington, DC, pp. 1-66.

Hadamard, J. S. 1911 Mouvement permanent lent d'une sphere liquide et visqueuse dans un liquide visqueux. C.R. Acad. Sci. Paris 152, 1735-1738.

Happel, J. \& Brenner, H. 1965 Low Reynolds Number Hydrodynamics. Prentice-Hall.

HaRTLAND, S. 1967 The approach of a liquid drop to a flat plate. Chem. Engng Sci. 22, 1675-1687.

HaRtLand, S. 1969 The profile of the draining film beneath a liquid drop approaching a plane interface. Chem. Engng Prog. Symp. Ser. no. 91, 65, 82-89.

Hartland, S. \& Robinson, J. D. 1977 A model for an axisymmetric dimpled draining film. J. Colloid Interface Sci. 60, 72-81.

Jones, A. F. \& WiLson, S. D. R. 1978 The film drainage problem in drop coalescence. J. Fluid Mech. 87, 263-288.

Kantorovich, L. V. \& KRYlov, V. I. 1963 Approximate Methods of Higher Analysis. Interscience.

LEe, S. H. \& Leal, L. G. 1982 The motion of a sphere in the presence of a deformable interface. II. A numerical study of the translation of a sphere normal to an interface. J. Colloid Interface Sci. 87, 81-106.

Lin, C. Y. \& Slattery, J. C. 1982 Thinning of a liquid film as a small drop or bubble approaches a solid plane. AIChE J. 28, 147-156.

ODqvist, F. K. G. 1930 Uber die Randwertaufgaben der Hydrodynamik Zaher Flussigkeiten. Math. Z. 32, 329-375.

OsEEN, C. W. 1927 Hydrodynamik. Akademische Verlagsgesellschaft.

Platikanov, D. 1964 Experimental investigation on the 'dimpling' of thin liquid films. J. Phys. Chem. 68, 3619-3624.

Pozrikidis, C. 1987 Creeping flow in two-dimensional channels. J. Fluid Mech. 180, 495-514.

Rallison, J. M. \& ACrivos, A. 1978 A numerical study of the deformation and burst of a viscous drop in an extensional flow. J. Fluid Mech. 89, 191-200.

Rybczynski, W. 1911 Uber die Fortschreitende Bewegung einer Flussigen Kugel in einem Zahen Medium. Bull. Acad. Sci. Cracovie (Ser. A), 40-46.

Tran-Cong, T. \& Phan-Thien, N. 1989 Stokes problems of multiparticle systems: a numerical method for arbitrary flows. Phys. Fluids A 1, 453-461.

YoungRen, G. K. \& ACRIVos, A. 1975 Stokes flow past a particle of arbitrary shape: a numerical method of solution. J. Fluid Mech. 69, 377-403 (and Corrigendum, 75 (1976) 813).

YOUNGREN, G. K. \& ACRIvos, A. 1976 On the shape of a gas bubble in a viscous extensional flow. J. Fluid Mech. 76, 433-442.

Zick, A. A. \& Homsy, G. M. 1982 Stokes flow through a periodic array of spheres. J. Fluid Mech. $115,13-26$. 